\title{
Effective bisector estimate with application to Apollonian circle packings
}

\author{
Ilya Vinogradov ${ }^{1}$
}

October 29, 2018

\footnotetext{
${ }^{1}$ Princeton University, Princeton, NJ
} 


\begin{abstract}
Let $\Gamma<\operatorname{PSL}(2, \mathbf{C})$ be a geometrically finite non-elementary discrete subgroup, and let its critical exponent $\delta$ be greater than 1 . We use representation theory of $\operatorname{PSL}(2, \mathbf{C})$ to prove an effective bisector counting theorem for $\Gamma$, which allows counting the number of points of $\Gamma$ in general expanding regions in $\operatorname{PSL}(2, \mathbf{C})$ and provides an explicit error term. We apply this theorem to give power savings in the Apollonian circle packing problem and related counting problems.
\end{abstract}




\section{Contents}

1. Introduction $\quad \mathbf{2}$

1.1 Lattice point counting problem. . . . . . . . . . . . . . . . . 2

1.2 Sector and bisector count. . . . . . . . . . . . . . . . . . . 2

1.3 Counting $\Gamma$-orbits in cones and hyperboloids. . . . . . . . . . . . . . 3

1.4 Acknowledgements. . . . . . . . . . . . . . . . . 5

2. Statement of results $\quad 5$

2.1 Bisector count. . . . . . . . . . . . . . . . . . 5

2.2 Application to Apollonian circle packing problem. . . . . . . . . . . . . . 9

3. Parametrization 11

3.1 The group G. . . . . . . . . . . . . . . . . . . . . . . . . . . . . . . . . . . . . . . . .

3.2 Hyperbolic space. . . . . . . . . . . . . . . . . . . . . . . . . . 12

4. Line model $\quad 13$

4.1 Complementary series for $G \ldots \ldots \ldots \ldots$. . . . . . . . . . . . . . . . . . . . . . . . .

4.2 Lie algebra of $G . \ldots \ldots \ldots$

4.3 Universal Enveloping Algebra of G. . . . . . . . . . . . . . . . . . 13

$4.4 \quad K$-type decomposition. . . . . . . . . . . . . . . . . . . . 14

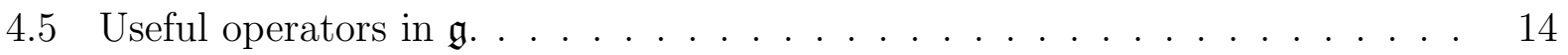

4.6 Normalizing operators. . . . . . . . . . . . . . . . . . . 16

5. Automorphic model $r$

5.1 Spectral decomposition. . . . . . . . . . . . . . . . . . . . . . . . . . . . . . .

5.2 Patterson-Sullivan theory. . . . . . . . . . . . . . . . . . . . . 18

5.3 Lie algebra elements in $K A^{+} K$ coordinates. . . . . . . . . . . . . . . . . . 19

6. Decay of matrix coefficients $\quad 20$

7. Proof of Theorem 2.1.2 21

7.1 Setup. . . . . . . . . . . . . . . . . . . . . 21

7.2 Spectral decomposition. . . . . . . . . . . . . . . . . . . . . 23

7.3 K-type decomposition. . . . . . . . . . . . . . . . . . 23

7.4 Main term. . . . . . . . . . . . . . . . . . . . . . . 24

7.5 Conjugation invariance. . . . . . . . . . . . . . . . 26

$7.6 K$ invariance. . . . . . . . . . . . . . . . . . . . . . . . . 29

7.7 Bounds in the variables $a$ and $a^{\prime} \ldots \ldots . \ldots . \ldots . . . \ldots 30$

8. Proof of Theorems 2.2.1 and 2.2.4 31

8.1 Setup. . . . . . . . . . . . . . . . . . . 31

8.2 Region of summation. . . . . . . . . . . . . . . . . . . . . . . . . . . . . . . . . . . . . 32

8.3 Smoothing. . . . . . . . . . . . . . . . . . . . . 33 
8.4 Applying Main Theorem. . . . . . . . . . . . . . . . . . . . . . . 33

8.5 Ideal triangle case. . . . . . . . . . . . . . . . . . . 35

\section{INTRODUCTION}

1.1 Lattice point counting problem. Let $G$ be a topological group with a norm $\|\cdot\|$ and let $\Gamma$ be a discrete subgroup. The question of estimating

$$
N(R)=\#\{\gamma \in \Gamma:\|\gamma\|<R\}
$$

as $R$ grows is known as the lattice point counting problem and was first asked by Gauss for $G=\mathbf{R}^{2},\|\cdot\|=\|\cdot\|_{L^{2}}$, and $\Gamma=\mathbf{Z}^{2}$; he showed that

$$
N(R)=\pi R^{2}+E(r)
$$

with $|E(r)|<2 \sqrt{2} \pi r$. The problem of improving the bound on the error function $E(r)$ is now called the Gauss circle problem. The current record $E(r)=O\left(r^{\sigma}\right)$ for $\sigma=\frac{131}{208}$ (ignoring logarithmic factors) is held by Huxley [17, 18], who improved previous results by van der Corput [38] $\left(\sigma=\frac{27}{41}\right)$, Vinogradov $\left(\sigma=\frac{34}{53}\right)$, and others.

Delsarte [4] and Huber [15, 16] initiated the study of this question in the hyperbolic setting: $G=\mathrm{SL}(2, \mathbf{R}), \Gamma$ a cocompact lattice, and the norm comes from the distance in $\mathbf{H}^{2}$. The difficulty that naturally arises here is that due to hyperbolic expansion: the measure of the boundary of the expanding ball grows roughly at the same rate as the measure of the ball itself. For this reason it is not clear at the first sight that the main term for $N(R)$ is given by the volume of the ball of radius $R$. Selberg [34] was able to produce an excellent error term in the function $N(R)$ for any lattice $\Gamma$ using his celebrated trace formula.

The question of understanding the growth of $N(R)$ for infinite covolume subgroups $\Gamma$ arose naturally. Patterson [32] and Sullivan [36, 37] developed extremely useful machinery for analyzing infinite covolume geometrically finite groups $\Gamma$ for $G=\operatorname{SL}(2, \mathbf{R})$ and more generally for $G=\mathrm{SO}(n, 1)$. To each such $\Gamma$ they associated a $\Gamma$-invariant probability measure on the boundary of the hyperbolic plane ( $n$-space) supported on the limit set of $\Gamma$, and related it to the spectrum of the Laplacian on the manifold $\Gamma \backslash \mathbf{H}$ when the base eigenvalue is at least $\left(\frac{n-1}{2}\right)^{2}$. Lax and Phillips [24] used wave equation techniques to produce a very good error term for the counting problem in $\mathbf{H}^{n}$ for general $n$ and showed that the spectrum of the Laplacian on $\Gamma \backslash \mathbf{H}^{n}$ has but finitely many non-tempered eigenvalues. The main order of growth in this case is related to the base eigenvalue $\delta(n-1-\delta)$ of the Laplacian on $\Gamma \backslash \mathbf{H}^{n}$ for some $\delta=\delta_{\Gamma} \in\left(\frac{n-1}{2}, n-1\right)$, and equals const $\cdot e^{\delta R}$ for the number of points in a ball of radius $R$. This is consistent with the fact that the volume of such ball grows like $e^{(n-1) R}$.

1.2 Sector and bisector count. A question which arises in applications of lattice point counting problem is counting in more general expanding sets, not just in balls. Both in the finite and infinite covolume case this problem can be approached by cutting a ball into sectors and using them to approximate a more general expanding set. In this setting the word bisector refers a subset $S$ of a semisimple Lie group $G=K A^{+} K$ (known as Cartan or polar decomposition) for which there exist contractible $S_{K_{1}} \subset K, S_{A} \subset A^{+}, S_{K_{2}} \subset K$ such 
that $S=S_{K_{1}} S_{A} S_{K_{2}}$. When $S_{K_{1}}=K=S_{K_{2}}$, the set $S$ is nothing but a ball. When one of $S_{K_{1}}, S_{K_{2}}$ is all of $K$, the set $S$ is referred to as a sector. In the case $G=\operatorname{SO}(n, 1)$ and $S_{K_{2}}=K$, the set $S_{K_{1}} A^{+} \subset G$ can be viewed as a subset of $\mathbf{H}^{n}=G / K$, and in the ball model this set will be a hyperbolic (and in fact Euclidean) sector, motivating these definitions.

In the case of lattices in $\operatorname{SL}(2, \mathbf{R})$ a very strong result was obtained by Good [9], and under additional assumptions of finite geometric property and critical exponent $\delta_{\Gamma}>\frac{1}{2}$ Bourgain, Kontorovich, and Sarnak [3] proved a similar counting statement without assuming that $\Gamma$ is a lattice. A very general asymptotic bisector counting theorem for lattices was proven by Gorodnik and Nevo [10]. Oh and Shah [29] proved an asymptotic bisector count for $G=\mathrm{SO}(n, 1)$ for non-lattices, but their proof relies on measure rigidity and is not readily made effective. In the present paper we prove an effective bisector counting theorem for $G=\mathrm{SO}(3,1)$ for non-lattices (under additional assumptions). Our approach is most similar to that of [3]: the main ingredient is representation theory of $L^{2}(\Gamma \backslash G)$. We develop explicit $K$-type decomposition for complementary series representations of PSL $(2, \mathbf{C})$ and then use the adjoint action of $G$ on conjugates of $\Gamma$ to avoid some computation.

The difficulty that arises in generalizing the current approach to $\mathrm{SO}(n, 1)$ is that parts of the argument are deeply rooted - albeit less so than that of [3] — in the structure of $K$ types for complementary series representations of $\mathrm{SO}(3,1)$. Many computations use specific forms of eigenfunctions, which is the main obstacle preventing direct generalization to higher dimensions. Nevertheless we anticipate that an effective bisector estimate should hold for $\mathrm{SO}(n, 1)$ for all $n \geqslant 4$.

There is another method that can be used to prove bisector theorems (and related limit theorems) in the case when $\Gamma \subset \mathrm{SO}(n, 1)$ is convex cocompact. In this case the problem can translated into the language of symbolic dynamics (cf. [23, 35]); we do not pursue this method here.

1.3 Counting $\Gamma$-orbits in cones and hyperboloids. Let $Q$ be a form defined over $\mathbf{Q}$. The problem of counting integer vectors $x \in \mathbf{Z}^{n}$ such that $Q(x)=c \in \mathbf{Z}$ for a given $c$ is very well studied. For definite diagonal forms it is related to Waring's problem [40]. For a general definite form one can ask for the growth rate of the number of solutions to $Q(x)=c$ as $c \rightarrow \infty$. In the case of an indefinite form one can fix $c$ such that $Q(x)=c$ has a solution, and ask for the growth rate of

$$
\#\{\|x\|<X \mid Q(x)=c\}
$$

as $X \rightarrow \infty$ for some norm $\|\cdot\|$ on $\mathbf{Z}^{n}$. When the number of variables $n$ is sufficiently large compared to the degree of the form then the problem may be solved by the Hardy-Littlewood circle method [39] for certain forms $Q$. Using different techniques Duke, Rudnick, and Sarnak [5] and Eskin and McMullen [7] proved that the number of solutions $\#\{\|x\|<X \mid Q(x)=c\}$ grows like the volume of the corresponding ball (under additional assumptions on $Q$ ).

In a similar fashion one can count solutions to $\#\{\|x\|<X \mid Q(x)=c\}$ with the additional constraint that $x$ lies in a prescribed orbit of a lattice $\Gamma<\mathrm{O}_{Q}(\mathbf{Z})$, but it is a harder question when $\Gamma$ is not a lattice. The bisector counting theorem allows us to compute $\#\left\{x \in \Gamma x_{0}:\|x\|<X\right\}$ with an explicit error term for $G=\mathrm{SO}(3,1)$ and certain groups non-lattices $\Gamma$. Note that the varieties $\{Q=c\}$ are fundamentally different according as $c$ is 


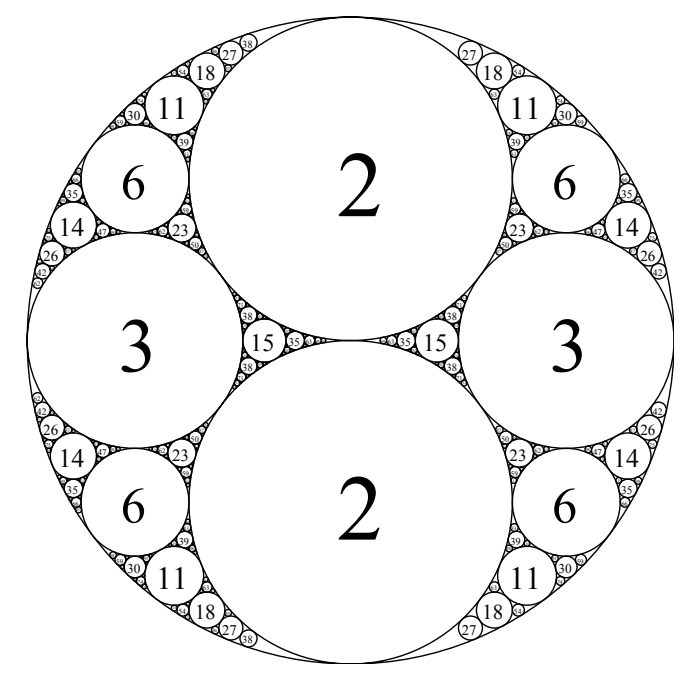

Figure 1.1: Bounded packing

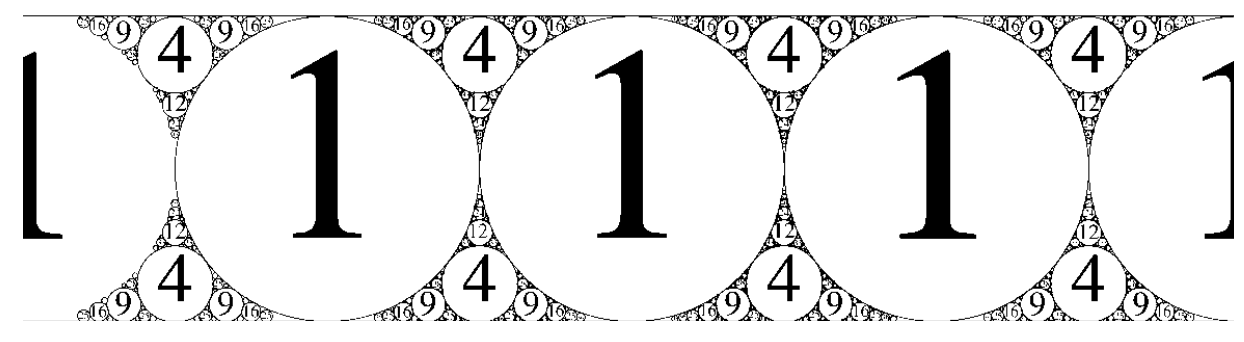

Figure 1.2: Unbounded packing

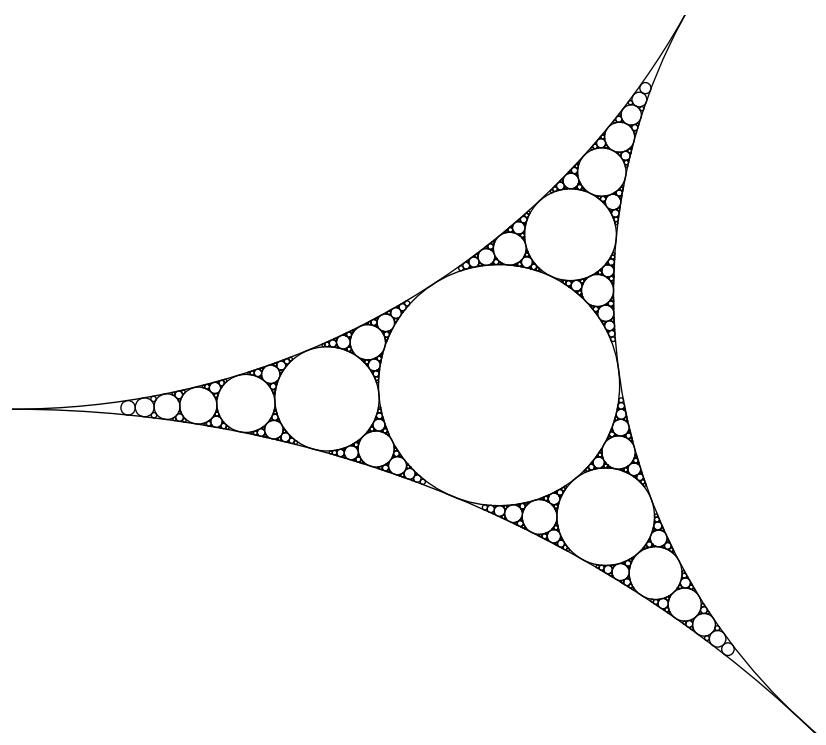

Figure 1.3: Apollonian packing in an ideal triangle. 
positive (one-sheeted hyperboloid), negative (two-sheeted hyperboloid), or zero (cone), but our approach works in every case.

A particular instance of this question is the Apollonian circle packing problem. Figures 1.1 and 1.2 show Apollonian circle packings. For a bounded packing $P$ let $N^{P}(T)$ be the number of circles in the packing having curvature at most $T$. For a periodic packing $N^{P}(T)$ is the number of such circles in one period. It has been shown $[11,21]$ that the $N^{P}(T)$ is related to counting points of the orbit of $\Gamma$ in the cone on the so-called Descartes form $Q_{D}$ which has signature $(3,1)$. The group $\Gamma$ in this case is the Apollonian group (up to finite index) which has infinite index in $\mathrm{O}_{Q_{D}}(\mathbf{Z})$. We show that

$$
N^{P}(T)=c_{P} T^{\delta}+O\left(T^{\delta-\varepsilon}\right)
$$

for constants $c_{P}, \delta$, and $\varepsilon$. The power savings depend on the spectral gap for $\Gamma$, which is not known numerically. We prove the same counting statement about the packing in Figure 1.3, which is known as the Apollonian gasket or the packing in an ideal triangle. Moreover we can prove effective versions for any circle packing theorem (not just the Apollonian one), such as the ones in [30] and [28].

1.4 Acknowledgements. I would like to acknowledge the help, inspiration, patience, and cheerful optimism of Alex Kontorovich, who introduced me to hyperbolic lattice point counting problems, guided me in every respect, kindly allowed me to use his pictures, and supported my travel through NSF Grant DMS-120937. I am greatly indebted to Yakov G. Sinai for acquainting me with dynamical problems in number theory as well as for his kindness and support. I greatly appreciate discussions with Peter Sarnak who was always supportive and persistent. I would also like thank Stephen Miller for supplying me with vital references.

I am very greatful to Ali Altuğ, Francesco Cellarosi, and Samuel Ruth for sharing the office with me and being always eager to answer my questions. I also thank Arul Shankar, Jonathan Luk, Mohammad Farajzadeh Tehrani, Kevin Hughes, Péter Varjú, and other graduate students who made my time at Princeton mathematically fruitful.

This manuscript would have been considerably less complete if it had not been scrutinized by Hee Oh and Nicolas Templier, whom I thank most kindly. My degree would have never been completed without the help of Jill LeClair, who makes simple that which is intricate.

Finally, I thank my wife, Milena Zhivotovskaya, and my family for their love and support.

\section{Statement of Results}

2.1 Bisector count. Let $\Gamma<\operatorname{PSL}(2, \mathbf{C})=G$ be a non-elementary geometrically finite discrete subgroup. The problem of counting the number of points in a $\Gamma$-orbit which lie in an expanding region in $G$ is well-studied in the case when $\Gamma \backslash G$ has finite volume $[34,9,5,7]$. The main term of such a count is always the volume of the expanding region. There is ample literature in the infinite volume case $[23,35,22,21,29]$. A major result in this setting is due to Lax and Phillips [24], who give an unsurpassed error term using a non-Euclidean wave equation. In the present paper we prove a more general counting theorem in the infinite volume case. 
Assume from now on that $\Gamma$ is no longer a lattice. The group $G$ naturally acts on $\mathbf{H}^{3}=\left\{x_{1}+i x_{2}+j y \mid x_{1}, x_{2} \in \mathbf{R}, y>0\right\}$ by Möbius transformations (isometries preserving the hyperbolic distance $d$ ) with quaternion multiplication, and the orbit $\Gamma j \subset \mathbf{H}^{3}$ is a discrete set. The set of its limit points $\Lambda_{\Gamma}$ in $\partial \mathbf{H}^{3}$ is known to be a Cantor set whose Hausdorff dimension we denote by $\delta$. As $\Gamma$ has infinite covolume we have $0<\delta<2$ [2]. The Patterson-Sullivan measure is a finite measure $\nu_{\Gamma}$ supported on $\Lambda_{\Gamma}$ which is a Hausdorff measure of dimension $\delta$. This measure is constructed (in essence) as the unique weak- $*$ limit of the family of measures

$$
\nu_{s, \Gamma}(z)=\frac{\sum_{\gamma \in \Gamma} e^{-s d(j, \gamma j)} \delta_{\gamma j}(z)}{\sum_{\gamma \in \Gamma} e^{-s d(j, \gamma j)}}
$$

as $s \rightarrow \delta$ from the right [32, 37, 36]. It is the unique finite measure (up to scalar multiples) with the property that

$$
\frac{d \gamma_{*} \nu_{\Gamma}}{d \nu_{\Gamma}}(\xi)=e^{-\delta \beta_{\xi}(\gamma j, j)}
$$

where $\beta_{\xi}\left(z_{1}, z_{2}\right)=\lim _{z \rightarrow \xi} d\left(z_{1}, z\right)-d\left(z_{2}, z\right)$ is the Busemann function and $\gamma_{*} \nu_{\Gamma}=\nu_{\Gamma} \circ \gamma^{-1}$ is the push-forward.

The group $G$ acts on $L^{2}(\Gamma \backslash G)$ : for $g \in G$ and $f \in L^{2}(\Gamma \backslash G)$ we have

$$
\text { g. } f(\Gamma x)=f(\Gamma x g)
$$

which is the right regular representation. Suppose $\delta>1$; then the hyperbolic Laplacian acts on the smooth functions inside this space and has an eigenfunction with eigenvalue $\lambda_{0}=\delta(2-\delta)<1$. It has but finitely many eigenvalues $\lambda_{n} \in(0,1)$ with $0 \leqslant n \leqslant d$, as was shown in [24]; arrange the eigenvalues in increasing order. We can write

$$
\lambda_{n}=s_{n}\left(2-s_{n}\right)
$$

for $1<s_{0}<s_{1} \leqslant \ldots s_{d}<2$ and decreasing in $n$. As a $G$-representation the right regular representation decomposes as

$$
V_{s_{0}} \oplus \cdots \oplus V_{s_{d}} \oplus V_{\text {temp }}
$$

where $V_{s_{n}}$ are isomorphic to complementary series representations with parameter $s_{n}$, and $s_{0}=\delta$.

In this setting Lax and Phillips [24] prove the following counting theorem.

Theorem 2.1.1 (Lax-Phillips). Assume $\delta>1$. Then

$$
\#\{\gamma \in \Gamma: d(j, \gamma j)<R\}=c_{0} e^{\delta R}+c_{1} e^{s_{1} R}+\cdots+c_{d} e^{s_{d} R}+O\left(e^{\frac{\delta+1}{2} R} R^{5}\right) .
$$

The constants $c_{n}$ are computed explicitly in terms of the corresponding eigenfunctions with eigenvalue $\lambda_{n}$.

For $g \in G$, let $k_{1}(g) a(g) k_{2}(g)$ be a $K A^{+} K$ decomposition; it is unique up to factors from $M$, which is the normalizer of $A$ in $K$. For the group $G$ in question we have

$$
K \cong \operatorname{PSU}(2), \quad A^{+} \cong \mathbf{R}^{+}, \quad M \cong \mathrm{SO}(2) .
$$


Thus, $K / M=S^{2}$ and $M \backslash K=S^{2}$ with different coordinates. Also define $K(g)$ to be $k_{1}(g) k_{2}(g)$. Notice that this definition is independent of the location of the $M$ factor.

There is an orthonormal basis for $L^{2}(K / M) \cong L^{2}\left(\partial \mathbf{H}^{3}\right)$ given by spherical harmonics $Y_{a b}(\varphi, \theta), a \geqslant 0,|b| \leqslant a$; we always assume that every compact group has measure 1 . For each fixed $a$ the collection $\left\{Y_{a b},|b| \leqslant a\right\}$ constitutes a basis for the $(2 a+1)$-dimensional irreducible representation of PSU(2). We can, and do, think of spherical harmonics as functions on $K$; in this case they are $M$-invariant on the right. More generally consider

$$
L^{2}(K)=\sum_{\tau \text { irrep of } K}(\operatorname{dim} \tau) \cdot \tau .
$$

Define generalized spherical harmonics

$$
Y_{a ; b c}\left(\varphi, \theta, \varphi_{2}\right)=e^{i b \varphi} y_{a ; b c}(\theta) e^{i c \varphi_{2}}
$$

to be an orthonormal basis for $L^{2}(K)$ such that for fixed $a$ and $c$ with $|c| \leqslant a$

$$
\left\{Y_{a ; b c}:|b| \leqslant a\right\}
$$

is a basis for the left regular representation of $K$ on $L^{2}(K)$; the usual spherical harmonics correspond to $c=0$. Writing

$$
Y_{a a^{\prime} ; b b^{\prime} c}\left(\varphi, \theta, \psi, \theta_{2}, \varphi_{2}\right)=Y_{a ; b c}(\varphi, \theta, 0) \overline{Y_{a^{\prime} ; b^{\prime} c}\left(\psi, \theta_{2}, \varphi_{2}\right)^{-1}}
$$

we have a basis for $L^{2}(K / M . K)$ given by

$$
\left\{Y_{a a^{\prime} ; b b^{\prime} c}\left(\varphi, \theta, \psi, \theta_{2}, \varphi_{2}\right):|b|,|c| \leqslant a ;\left|b^{\prime}\right|,|c| \leqslant a^{\prime}\right\} .
$$

This basis inherits both left and right transformation properties under $K$. We can write $Y_{a a^{\prime} ; b b^{\prime} c}(K(g))$ using our coordinates; whenever $c=0$ we have

$$
Y_{a a^{\prime} ; b b^{\prime} c}(K(g))=Y_{a b}\left(k_{1}(g)\right) \overline{Y_{a^{\prime} b^{\prime}}\left(k_{2}^{-1}(g)\right)} .
$$

Finally let $|\cdot|$ be a bi- $K$-invariant norm on $G$ normalized as explained in (3.1.2) below. We prove the following theorem.

Theorem 2.1.2. Assume $\delta>1$. Let

$$
0<\delta(2-\delta)=\lambda_{0}<\lambda_{1} \leqslant \ldots \leqslant \lambda_{d}<1
$$

be the eigenvalues below 1 of the hyperbolic Laplacian on $\Gamma \backslash \mathbf{H}^{3}$. Then

$$
\begin{aligned}
& \sum_{\gamma \in \Gamma,|\gamma|<T} Y_{a^{\prime} a ; b^{\prime} b c}(K(\gamma))=\frac{\pi \mathbf{1}_{\{c=0\}} T^{2 \delta}}{\delta(\delta-1)} \int_{K / M} Y_{a b}(k) d \nu_{\Gamma}(k) \int_{K / M} Y_{a^{\prime} b^{\prime}}(k) d \nu_{\Gamma}(k)+ \\
& +\mathbf{1}_{\{c=0\}} c_{1}\left(a, b, a^{\prime}, b^{\prime}\right) T^{2 s_{1}}+\cdots+1_{\{c=0\}} c_{d}\left(a, b, a^{\prime}, b^{\prime}\right) T^{2 s_{d}}+ \\
& +O\left(T^{2 \frac{10 \delta+1}{11}}(\log T)^{1 / 11}(a+1)^{15 / 11}\left(a^{\prime}+1\right)^{15 / 11}\right) .
\end{aligned}
$$

The numbers $s_{1}, \ldots, s_{d}$ are less than $\delta$ and $>1$ and satisfy $s_{n}\left(2-s_{n}\right)=\lambda_{n}$. Additionally,

$$
\left|c_{n}\left(a, b, a^{\prime}, b^{\prime}\right)\right| \ll\left((a+1)\left(a^{\prime}+1\right)\right)^{2-s_{n}+\frac{1}{2}}\left|c_{n}(0,0,0,0)\right|,
$$

and the implied constant depend only on $\Gamma$. 
Remark 2.1.3. The Patterson-Sullivan [32,37] measure in the statement is normalized so that the corresponding base eigenfunction of the Casimir operator has $L^{2}$ norm 1 ; we explain this in (5.2.1).

Remark 2.1.4. The special case with trivial spherical harmonics matches the main term Theorem 2.1.1, but the error term in our treatment is worse.

Remark 2.1.5. The Theorem implies that there are cancellations when $c$ is non-zero. The most interesting case, i.e., the case in which we compute the main term, is $c=0$. We can rewrite it more succinctly as

$$
\begin{aligned}
\sum_{\gamma \in \Gamma,|\gamma|<T} Y_{a^{\prime} b^{\prime}}\left(k_{1}(\gamma)\right) \overline{Y_{a b}\left(k_{2}^{-1}(\gamma)\right)} & =\frac{\pi T^{2 \delta}}{\delta(\delta-1)} \int_{K / M} Y_{a b}(k) d \nu_{\Gamma}(k) \int_{K / M} Y_{a^{\prime} b^{\prime}}(k) d \nu_{\Gamma}(k)+ \\
& +c_{1}\left(a, b, a^{\prime}, b^{\prime}\right) T^{2 s_{1}}+\cdots+c_{d}\left(a, b, a^{\prime}, b^{\prime}\right) T^{2 s_{d}}+ \\
& +O\left(T^{2 \frac{10 \delta+1}{11}}(\log T)^{1 / 11}(a+1)^{15 / 11}\left(a^{\prime}+1\right)^{15 / 11}\right) .
\end{aligned}
$$

Remark 2.1.6. The Theorem is only interesting when $T$ is sufficiently large depending on $a$, $a^{\prime}$. If the error term dominates the main term a better bound is obtained from Theorem 2.1.1 by observing that

$$
\left\|Y_{a^{\prime} a ; b^{\prime} b c}\right\|_{L^{\infty}} \ll \sqrt{(a+1)\left(a^{\prime}+1\right)}
$$

whence the right hand side of (2.1.1) can be replaced by

$$
\ll T^{2 \delta} \sqrt{(a+1)\left(a^{\prime}+1\right)} .
$$

This theorem is a generalization of the work of Bourgain, Kontorovich, and Sarnak in [3] for $\operatorname{SL}(2, \mathbf{R})$; we generalize parts of their method to $\operatorname{PSL}(2, \mathbf{C})$. In the special case $c=0$, the main term has been obtained by Oh and Shah [29], but their method uses measure rigidity and therefore is not readily made effective. In the present paper we use spectral theory of $L^{2}(\Gamma \backslash G)$, which provides explicit error terms. For the same reason the method of the present paper cannot be applied once $\delta \leqslant 1$ as there are no $L^{2}$ eigenfunctions on $\Gamma \backslash \mathbf{H}^{3}$, but Oh and Shah give asymptotics in that case, too.

The proof of the Main Theorem relies on spectral theory, but we do not follow the original treatment of [3]. We do not attempt to compute the leading term for every combination $a, b, a^{\prime}, b^{\prime}, c$ directly - although it is probably possible, and we give tools adequate for computing the main term in any particular case - but instead develop a new method using conjugates of $\Gamma$ to obtain the statement for general indices; full computation is only required in one case. This new approach works inductively on the indices and relies on the observation that once Theorem 2.1.2 is established for one fixed set of indices and all suitable subgroups $\Gamma$, the validity of Theorem 2.1.2 can be extended other sets of indices using the freedom in $\Gamma$. If $g=\exp \varepsilon X \in G$ for some $X \in \mathfrak{g}$ is close to the identity we can apply Theorem 2.1.2 to $\Gamma_{g}=g \Gamma g^{-1}$ and linearize equation (2.1.1) in $\varepsilon$. Equation (2.1.1) for other choices of indices can then be recovered as equality between lower order terms for suitably chosen $X$. 
A simplified weaker version of Theorem 2.1.2 will be useful to us. Under the assumptions of the Theorem, equation (2.1.3) can be replaced by

$$
\begin{aligned}
& \sum_{\gamma \in \Gamma,|\gamma|<T} Y_{a^{\prime} b^{\prime}}\left(k_{1}(\gamma)\right) \overline{Y_{a b}\left(k_{2}^{-1}(\gamma)\right)}=\frac{\pi T^{2 \delta}}{\delta(\delta-1)} \overline{\int_{K / M} Y_{a b}(k) d \nu_{\Gamma}(k)} \int_{K / M} Y_{a^{\prime} b^{\prime}}(k) d \nu_{\Gamma}(k)+ \\
& +O\left(T^{2 \frac{10 \delta+s_{1}}{11}+\varepsilon}(a+1)^{15 / 11}\left(a^{\prime}+1\right)^{15 / 11}\right) .
\end{aligned}
$$

In this version we incorporate the lower order main terms into the error term.

2.2 Application to Apollonian circle packing problem. Figures 1.1 and 1.2 show integral Apollonian circle packings. A thorough introduction to this subject can be found in [11]. For a bounded packing $P$ let $N^{P}(T)$ be the number of circles in the packing having curvature at most $T$. For a periodic packing $N^{P}(T)$ is the number of such circles in one period.

The growth of this function of $T$ was analyzed by Kontorovich and Oh [21] who proved the asymptotic formula

$$
N^{P}(T) \sim c_{P} T^{\delta}
$$

Here $c_{P}$ is some constant depending on $P$ and $\delta$ is the Hausdorff dimension of the packing. Since any packing can be sent to any other by a Möbius transformation, the dimension is a universal constant $\delta$, which McMullen computed to be $\approx 1.30568$ [27]. The approach of [21] is to relate the above problem to counting lattice points in the unit tangent bundle of an infinite volume hyperbolic 3 -manifold $\Gamma \backslash T^{1} \mathbf{H}^{3}$, where $\Gamma$ is a finite index subgroup of the Apollonian group (see $[21,11]$ ).

Let $Q_{D}$ be the Descartes form

$$
Q_{D}(a, b, c, d)=a^{2}+b^{2}+c^{2}+d^{2}-\frac{1}{2}(a+b+c+d)^{2}
$$

it has signature $(3,1)$. To each packing one can associate $v \in \mathbf{R}^{4}$ with $Q_{D}(v)=0$. The four entries of $v$ are the curvatures of the four circles (with usual conventions regarding signs and orientation) that generate the packing as an orbit of $v$ under the action of $\Gamma$. Let $v=g u=g(0,0,1,1)^{T}$ for some $g \in \mathrm{O}_{Q_{D}}(\mathbf{R})$ and let $\mathrm{B}=g^{-1} \Gamma g$; also let $\mathrm{B}^{\prime}=\left(S_{1} g\right)^{-1} \Gamma\left(S_{1} g\right)$ where $S_{1}$ is a generator of the Apollonian group (8.1.3).

In this setting we use Theorem 2.1.2 to prove power savings in the Apollonian circle packing problem and compute the overall constant ${ }^{1}$.

Theorem 2.2.1. For every $\varepsilon>0$ we have

$$
N^{P}(T)=c_{P} T^{\delta}+O_{\varepsilon}\left(T^{\frac{128 \delta+s_{1}}{129}+\varepsilon}\right) .
$$

\footnotetext{
${ }^{1}$ Added in print: Lee and $\mathrm{Oh}[25]$ have independently obtained a version of this statement using different methods.
} 
Remark 2.2.2. The constant $c_{P}$ can be expressed in terms of Patterson-Sullivan measure as

$$
\begin{aligned}
c_{P}=\frac{\pi}{\delta(\delta-1)} \int_{(\mathrm{B} \cap N) \backslash \partial \mathbf{H}^{3}}\left(|z|^{2}\right. & +1)^{\delta} d \nu_{\mathrm{B}}(z) \int_{\partial \mathbf{H}^{3}} \frac{d \nu_{\mathrm{B}}(k)}{\|g k u\|_{L^{\infty}}^{\delta}}+ \\
& +\frac{\pi}{\delta(\delta-1)} \int_{\left(\mathrm{B}^{\prime} \cap N\right) \backslash \partial \mathbf{H}^{3}}\left(|z|^{2}+1\right)^{\delta} d \nu_{\mathrm{B}^{\prime}}(z) \int_{\partial \mathbf{H}^{3}} \frac{d \nu_{\mathrm{B}^{\prime}}(k)}{\left\|S_{1} g k u\right\|_{L^{\infty}}^{\delta}} .
\end{aligned}
$$

Remark 2.2.3. The constant in front of the main term of this expression is several notation changes away from the similar expression in [21]. We use column vectors $v$ instead of row vectors, related by transposition. The quantity $g$ from the present paper corresponds to $\left(g_{0}^{-1}\right)^{T}$ in [21], and $u$ is $v_{0}^{T}$.

More generally let $\vartheta: \operatorname{PSL}(2, \mathbf{C}) \rightarrow \mathrm{SO}_{Q}^{\circ}(\mathbf{R})$ be a fixed isomorphism (used implicitly) for a quadratic form $Q$ of signature $(3,1)$. Let $\Gamma<\mathrm{SO}_{Q}^{\circ}(\mathbf{R})$ be a discrete subgroup subject to the hypotheses of Theorem 2.1.2. Let $u \in\{Q=0\}$ be such that Stab $u=N M$, let $v=g u$, and let $\mathrm{B}=g^{-1} \Gamma g$. Also let $\|\cdot\|$ be a norm on $\mathbf{R}^{4}$.

Theorem 2.2.4. For every $\varepsilon>0$ we have

$$
\begin{array}{r}
\sum_{\substack{\|\gamma v\|<T \\
\gamma \in \Gamma}} \chi_{G /(\operatorname{Stab} v \cap \Gamma)}(\gamma)=\left[\frac{\pi}{\delta(\delta-1)} \int_{(\mathrm{B} \cap N) \backslash \partial \mathbf{H}^{3}}\left(|z|^{2}+1\right)^{\delta} d \nu_{\mathrm{B}}(z) \int_{\partial \mathbf{H}^{3}} \frac{d \nu_{\mathrm{B}}(k)}{\|g k u\|^{\delta}}\right] \\
+O_{\varepsilon}\left(T^{\frac{128 \delta+s_{1}}{129}+\varepsilon}\right) .
\end{array}
$$

Remark 2.2.5. In fact $\|\cdot\|$ only needs to satisfy the scaling property of the norm together with non-vanishing and continuity; the full power of the triangle inequality is not needed.

Remark 2.2.6. We can assume that $v \in\{Q=c\}$ for some $c$ other than zero; a similar result holds in this case, but then $\operatorname{Stab} v \cong K$ or $\operatorname{Stab} v \cong \mathrm{SO}^{\circ}(2,1)$.

Theorem 2.1.2 allows for a further refinement of the Apollonian circle packing problem. Consider the packing in an ideal triangle as shown in Figure 1.3. It is contained in the region bounded by three tangent circles as shown; it is also contained in the circle through the vertices of the triangle. Let $S_{4}$ denote reflection in this circle and let $v \in \mathbf{R}^{4}$ consist of the curvatures of the four largest circles of the packing ordered so that $S_{4}$ preserves the three largest circles. Let $G_{4}$ be the disk bounded by the circumscribed circle of the triangle.

Theorem 2.2.7. With notation as in Theorem 2.2.1 we have

$$
N^{P}(T)=c_{P} T^{\delta}+O_{\varepsilon}\left(T^{\frac{128 \delta+s_{1}}{129}+\varepsilon}\right)
$$

where

$$
\begin{aligned}
c_{P}=\frac{\pi}{\delta(\delta-1)} \int_{G_{4}}\left(|z|^{2}+1\right)^{\delta} d \nu_{\mathrm{B}}(z) \int_{\partial \mathbf{H}^{3}} \frac{d \nu_{\mathrm{B}}(k)}{\|g k u\|_{L^{\infty}}^{\delta}}+ \\
+\frac{\pi}{\delta(\delta-1)} \int_{G_{4}}\left(|z|^{2}+1\right)^{\delta} d \nu_{\mathrm{B}^{\prime}}(z) \int_{\partial \mathbf{H}^{3}} \frac{d \nu_{\mathrm{B}^{\prime}}(k)}{\left\|S_{1} g k u\right\|_{L^{\infty}}^{\delta}} .
\end{aligned}
$$




\section{PARAMETRIZATION}

In this section we recall classical facts about the hyperbolic space and related Lie groups.

3.1 The group $G$. The group $\operatorname{PSL}(2, \mathbf{C})$ is realized as the group of $2 \times 2$ matrices with complex values having determinant 1 , modulo the center $\{ \pm 1\}$. This group is isomorphic to $\mathrm{SO}^{\circ}\left(x^{2}+y^{2}+z^{2}-w^{2}\right) \cong \mathrm{SO}^{\circ}(3,1)$. An explicit map from $\mathrm{PSL}(2, \mathbf{C})$ to $\mathrm{SO}^{\circ}\left(x^{2}+y^{2}+z^{2}-w^{2}\right)$ is given by

$$
\begin{aligned}
& \iota: \pm 2\left(\begin{array}{ll}
a & b \\
c & d
\end{array}\right) \mapsto \\
& \left(\begin{array}{cccc}
|a|^{2}+|d|^{2}-|b|^{2}-|c|^{2} & b \bar{a}+a \bar{b}-d \bar{c}-c \bar{d} & i(b \bar{a}-a \bar{b}-d \bar{c}+c \bar{d})|a|^{2}+|b|^{2}-|c|^{2}-|d|^{2} \\
c \bar{a}-d \bar{b}+a \bar{c}-b \bar{d} & d \bar{a}+c \bar{b}+b \bar{c}+a \bar{d} & i(d \bar{a}-c \bar{b}+b \bar{c}-a \bar{d}) & c \bar{a}+d \bar{b}+a \bar{c}+b \bar{d} \\
i(-c \bar{a}+d \bar{b}+a \bar{c}-b \bar{d}) & i(-d \bar{a}-c \bar{b}+b \bar{c}+a \bar{d}) d \bar{a}-c \bar{b}-b \bar{c}+a \bar{d} & i(-c \bar{a}-d \bar{b}+a \bar{c}+b \bar{d}) \\
|a|^{2}+|c|^{2}-|b|^{2}-|d|^{2} & b \bar{a}+a \bar{b}+d \bar{c}+c \bar{d} & i(b \bar{a}-a \bar{b}+d \bar{c}-c \bar{d})|a|^{2}+|b|^{2}+|c|^{2}+|d|^{2}
\end{array}\right)
\end{aligned}
$$

(cf. [11]). We suppress $\iota$ if no confusion can arise.

In this realization of $\mathrm{SO}(3,1)=\mathrm{SO}\left(x^{2}+y^{2}+z^{2}-w^{2}\right)$ take the norm $|\cdot|$ to be

$$
|g|=|a(g)|=\left|\left(\begin{array}{cccc}
\operatorname{ch} t & & & \operatorname{sh} t \\
& 1 & & \\
& & 1 & \\
\operatorname{sh} t & & & \operatorname{ch} t
\end{array}\right)\right|=e^{t} .
$$

It corresponds to

$$
\left|\left(\begin{array}{cc}
e^{t / 2} & \\
& e^{-t / 2}
\end{array}\right)\right|=\max \left(e^{t / 2}, e^{-t / 2}\right)
$$

on $\operatorname{PSL}(2, \mathbf{C})$. Therefore the statement of Theorem 2.1.2 for $\mathrm{SO}(3,1)$ would have $T^{2}$ replaced by $T$ on the right hand side.

We set

$$
\begin{aligned}
\Theta & =\left(\begin{array}{ll} 
& 1 \\
-1 &
\end{array}\right), \quad \Phi=\left(\begin{array}{ll}
i & \\
& -i
\end{array}\right) \in \mathfrak{s u}(2, \mathbf{C}), \quad h=\left(\begin{array}{ll}
1 & \\
& -1
\end{array}\right) \in \mathfrak{s l}(2, \mathbf{C}) \\
A^{+} & =\left\{\left(\begin{array}{ll}
e^{t / 2} & \\
& e^{-t / 2}
\end{array}\right): t \geqslant 0\right\}=\{\exp (t h / 2): t \geqslant 0\}, \\
N & =\left\{\left(\begin{array}{ll}
1 & z \\
& 1
\end{array}\right): z \in \mathbf{C}\right\} \\
M & =\left\{\left(\begin{array}{cc}
e^{i \varphi / 2} & e^{-i \varphi / 2}
\end{array}\right): \varphi \in[0,2 \pi)\right\} \\
K & =\left\{\exp (\varphi \Phi / 2) \exp (\theta \Theta / 2) \exp \left(\varphi_{2} \Phi / 2\right): \varphi \in[0, \pi), \varphi_{2} \in[0,2 \pi), \theta \in[0, \pi)\right\} .
\end{aligned}
$$

The latter parametrization of $K$ is called the Euler angle parametrization. The Haar measure on $K=\mathrm{PSU}(2)$ in these coordinates is given by

$$
d k=\frac{1}{4 \pi^{2}} \sin \theta d \varphi d \theta d \varphi_{2},
$$


and the Haar measure on $A^{+}$is

$$
d a_{t}=\operatorname{sh}^{2} t d t
$$

On the manifold $K / M$ we get the measure

$$
d k=\frac{1}{4 \pi} \sin \theta d \varphi d \theta
$$

we will use $d k$ to denote the measure both on $K / M$ and $K$. Thus we have almost everywhere unique decomposition

$$
g=\exp (\varphi \Phi / 2) \cdot \exp (\theta \Theta / 2) \cdot \exp (\psi \Phi / 2) \cdot \exp (t h / 2) \cdot \exp \left(\theta_{2} \Theta / 2\right) \cdot \exp \left(\varphi_{2} \Phi / 2\right) .
$$

The Haar measure on $\operatorname{PSL}(2, \mathbf{C})$ in these coordinates is

$$
4 \pi d k_{1} d a_{t} d k_{2}
$$

Here $d k_{1}$ is the probability measure on $K / M$, and $d k_{2}$ is the probability measure on $K$. The normalization of (3.1.5) is actually quite natural in view of the relation of $G$ to hyperbolic space, as we explain below.

3.2 Hyperbolic space. We use two models for $\mathbf{H}^{3}$, the upper half plane and the ball model (see [6] for details). The upper half space model is realized as $\left\{x_{1}+i x_{2}+j y \mid x_{1}, x_{2} \in \mathbf{R}, y>\right.$ $0\}$. The hyperbolic metric and volume are

$$
d s^{2}=\frac{d x_{1}^{2}+d x_{2}^{2}+d y^{2}}{y^{2}} \quad d V=\frac{d x_{1} d x_{2} d y}{y^{3}} .
$$

The ball model $\mathbf{B}^{3}$ is realized as the unit ball $\left\{x_{1}+i x_{2}+j y: x_{1}^{2}+x_{2}^{2}+y^{2}<1\right\} \subset \mathbf{R}^{3}$. We use the isomorphism

$$
z \mapsto(z-j)(-j z+1)^{-1}
$$

from $\mathbf{H}^{3}$ to $\mathbf{B}^{3}$ to transfer the metric. In these coordinates the metric and volume elements are

$$
d s^{2}=4 \frac{d x_{1}^{2}+d x_{2}^{2}+d y^{2}}{\left(1-x_{1}^{2}-x_{2}^{2}-y^{2}\right)^{2}} \quad d V=8 \frac{d x_{1} d x_{2} d y}{\left(1-x_{1}^{2}-x_{2}^{2}-y^{2}\right)^{2}} .
$$

We also identify boundaries $\partial \mathbf{H}^{3}$ and $S^{2} \cong K / M$ by extending the map there.

In the ball model there is a convenient set of coordinates that match the action of $G$. Any point in $\mathbf{B}^{3}=G / K$ is the image of 0 under an element of $A^{+}$and an element of $K / M$. To wit,

$$
\begin{aligned}
\left(\begin{array}{ll}
e^{i \varphi / 2} & \\
& e^{-i \varphi / 2}
\end{array}\right)\left(\begin{array}{cc}
\cos \frac{\theta}{2} & \sin \frac{\theta}{2} \\
-\sin \frac{\theta}{2} & \cos \frac{\theta}{2}
\end{array}\right)\left(\begin{array}{ll}
e^{t / 2} & \\
& e^{-t / 2}
\end{array}\right) 0= \\
=\operatorname{th} \frac{t}{2}(-\cos \varphi \sin \theta-i \sin \varphi \sin \theta+j \cos \theta) .
\end{aligned}
$$

In these coordinates the volume element reads

$$
d V=\sin \theta d \varphi d \theta \cdot \mathrm{sh}^{2} t d t=4 \pi d k_{1} d a_{t} .
$$

This measure extends to all of $G$ as in (3.1.5). The advantage of these normalizations is that respect the structure of $G$ as a $K$-bundle over $\mathbf{H}$. In particular, the natural map $L^{2}(\mathbf{H}) \rightarrow L^{2}(G)$ is an isometry. 


\section{LINE MODEL}

The goal of this section is to understand the action of $\mathfrak{g}^{\mathbf{C}}$ on the $K$-types of a complementary series representation. Neither the formula from Lemma 4.6.4 nor the action of certain operators on the $K$-types (4.6.4), (4.6.6), (4.6.5) is widely available in the literature. Operators similar to those we introduce appeared in [19] without normalizations.

4.1 Complementary series for $G$. Here we review the complementary series representations of $G$ in the line model $[20,8]$. Let $0<s<2$ and let $G$ act on functions $f: \mathbf{C} \rightarrow \mathbf{C}$ by

$$
\pi_{s}\left(\begin{array}{ll}
a & b \\
c & d
\end{array}\right) \cdot f(z)=\frac{1}{|b z+d|^{2 s}} f\left(\frac{a z+c}{b z+d}\right) .
$$

Denote by $I$ the intertwining operator

$$
I . f(\zeta)=\int_{\mathbf{C}} \frac{f(z)}{|z-\zeta|^{2(2-s)}} \frac{i}{2} d z \wedge d \bar{z} .
$$

The action above gives a unitary representation of $G$ on the space of functions from $\mathbf{C}$ to $\mathbf{C}$ equipped with the inner product

$$
\left\langle f_{1}, f_{2}\right\rangle=\int_{\mathbf{C}} f_{1}(z) \overline{I \cdot f_{2}(z)} \frac{i}{2} d z \wedge d \bar{z} ;
$$

call this space $V_{s}$. Then the complementary series representation of $G$ with parameter $s$ is $\left(\pi_{s}, V_{s}\right)$; it is non-tempered and irreducible.

4.2 Lie algebra of $G$. We parametrize the (real six-dimensional) Lie algebra $\mathfrak{g}=\mathfrak{s l}(2, \mathbf{C}) \cong$ $\mathfrak{s o}(3,1)$ by

$$
h=\left(\begin{array}{ll}
1 & \\
& -1
\end{array}\right) \quad e=\left(\begin{array}{l}
1 \\
\end{array}\right) \quad f=\left(\begin{array}{l} 
\\
1
\end{array}\right)
$$

and

$$
i h=\left(\begin{array}{ll}
i & \\
& -i
\end{array}\right) \quad \text { ie }=\left(\begin{array}{l}
i \\
\end{array}\right) \quad \text { if }=\left(\begin{array}{l} 
\\
i
\end{array}\right) .
$$

Note that the letter " $i$ " is concatenated to the symbol it follows.

4.3 Universal Enveloping Algebra of $G$. Let $\mathfrak{g}^{\mathbf{C}}$ denote the complexification of $\mathfrak{g}$; it is a complex six-dimensional Lie algebra whose field of scalars $\mathbf{C}$ we write as $a+b \mathrm{I}, a, b \in \mathbf{R}$. As usual, we denote its universal enveloping algebra by $U\left(\mathfrak{g}^{\mathbf{C}}\right)$ and lift the representation $\pi_{s}$ to it. We shall indulge in the sin of referring to $\pi_{s}$ in this way whether it is a representation of $G, \mathfrak{g}$, or $U\left(\mathfrak{g}^{\mathbf{C}}\right)$; we shall also drop the subscript $s$ in most cases.

The center $Z$ of $U\left(\mathfrak{g}^{\mathbf{C}}\right)$ is one-dimensional and is generated by the element

$$
\Omega=h^{2}-i h^{2}+2(e f+f e-i e i f-i f i e) ;
$$

it is called the Casimir operator and has the property that

$$
\pi(\Omega) \cdot F+4 s(2-s) F=0
$$

for smooth $F \in V_{s}$. 
4.4 $K$-type decomposition. Consider the decomposition of $\left(\pi_{s}, V_{s}\right)$ into $K$ types. That is, restrict $\pi$ to $K<G$ and decompose $V_{s}$ into irreducible representations of $K$. The group $K$ has one irreducible representation in every odd dimension. The multiplicity of each irreducible representation in $\left.\pi\right|_{K}$ is 1 . Let $\left\{v_{l j}^{s}: l \geqslant 0,|j| \leqslant l\right\}$ be an orthonormal basis for $V_{s}$ subject to the conditions

$$
\begin{gathered}
\pi\left(\Omega_{K}\right) v_{l j}^{s}+l(l+1) v_{l j}^{s}=0 \\
\pi(i h) v_{l j}^{s}=2 i j v_{l j}^{s},
\end{gathered}
$$

where $\Omega_{K}=\frac{1}{4}\left(i h^{2}+(i e+i f)^{2}+(e-f)^{2}\right)$ is the Casimir element for $K$. Thus for each $l$ the set $\left\{v_{l j}^{s}:|j| \leqslant l\right\}$ is a basis for the $(2 l+1)$-dimensional irreducible representation of $K$. This allows one to recover exact formulae for $v_{l j}^{s}$ as solutions to the system of ordinary differential equations (4.4.1)-(4.4.2). We will suppress the index $s$ if no confusion can arise.

4.5 Useful operators in $\mathfrak{g}$. The following elements of $U\left(\mathfrak{g}^{\mathbf{C}}\right)$ will be important for our investigation:

$$
\begin{aligned}
J^{ \pm} & =f \pm \mathrm{I} i f \\
R & =i e+i f+\mathrm{I}(e-f) \\
L & =i e+i f-\mathrm{I}(e-f) .
\end{aligned}
$$

The operators $R$ and $L$ (raising and lowering, resp.) are the usual ladder operators for $\mathfrak{s u}(2)$ and map $v_{l j}$ to multiples of $v_{l, j+1}$ and $v_{l, j-1}$, respectively.

Lemma 4.5.1. The action of $J^{ \pm}$satisfies

$$
\pi\left(J^{+}\right) v_{l l}=C(l) v_{l+1, l+1}
$$

and

$$
\pi\left(J^{-}\right) v_{l,-l}=C(l) v_{l+1,-l-1}
$$

for certain normalizing constants $C(l)$.

Proof. We only prove the first statement; the proof of the second one is similar.

The computation lies entirely in the Lie algebra. Define the following elements of $\mathfrak{s u}(2)$ :

$$
X=\left(\begin{array}{ll}
i & \\
& -i
\end{array}\right) \quad Y=\left(\begin{array}{rr} 
& 1 \\
-1 &
\end{array}\right) \quad Z=\left(\begin{array}{ll} 
& i \\
i &
\end{array}\right)
$$

and the following elements from $\mathfrak{s l}(2, \mathbf{C})$ :

$$
i X=\left(\begin{array}{ll}
-1 & \\
& 1
\end{array}\right) \quad i Y=\left(\begin{array}{cc} 
& i \\
-i &
\end{array}\right) \quad i Z=\left(\begin{array}{ll}
-1 \\
-1
\end{array}\right) .
$$

The Casimir operator for $\mathfrak{s u}(2)$ can be written as

$$
\Omega_{K}=\frac{1}{4}\left(X^{2}+Y^{2}+Z^{2}\right)
$$


With this normalization we have

$$
\pi\left(\Omega_{K}\right) v_{l j}=-l(l+1) v_{l j}
$$

for every $|j| \leqslant l$. Thus to claim that $f+$ Iif is the correct operator we need two conditions to hold:

$$
\pi\left(\left[\Omega_{K}, f+\mathrm{I} i f\right]\right) v_{l l}=-2(l+1) v_{l l}
$$

and

$$
\pi([X / 2, f+\mathrm{I} i f]) v_{l l}=\mathrm{I}(f+\mathrm{I} \text { if }) v_{l l} .
$$

This first condition ensures that the Casimir eigenvalue changes from $-l(l+1)$ to $-(l+$ $1)(l+2)$, while the second condition guarantees that the eigenvalue of $X / 2$ is increased by I. Since we assume we start with $v_{l l}$, our initial assumptions are

$$
\begin{aligned}
\pi\left(\Omega_{K}\right) v_{l l} & =-l(l+1) v_{l l}, \\
\pi(X) v_{l l} & =2 \mathrm{I} l v_{l l}, \\
\pi(R) v_{l l} & =\pi(Z+\mathrm{I} Y) v_{l l}=0 .
\end{aligned}
$$

Thus we compute

$$
\begin{aligned}
& {\left[\Omega_{K}, f+\mathrm{I} i f\right]=} \\
& =\left[\frac{X^{2}+Y^{2}+Z^{2}}{4}, \frac{-Y-i Z+\mathrm{I} Z-\mathrm{I} i Y}{2}\right]=\frac{1}{2}(X(i Y-\mathrm{I} i Z)-i X(Y-\mathrm{I} Z))= \\
& =\frac{1}{2}(i X \mathrm{I}(Z+\mathrm{I} Y)+(i Y-\mathrm{I} i Z) X+[X, i Y-\mathrm{I} i Z]) .
\end{aligned}
$$

The first term contains the raising operator $Z+\mathrm{I} Y$, so we drop it. The second term contains $X$, which we replace by $2 \mathrm{I} l$. The last term we massage further to get

$$
(l+1)(i Z+\mathrm{I} i Y)
$$

Recalling that $\pi(Z+\mathrm{I} Y)=0$ on $v_{l l}$, we can write (4.5.10) as

$$
-2(l+1) \frac{-Y-i Z+\mathrm{I} Z-\mathrm{I} i Y}{2},
$$

confirming (4.5.5). The second condition (4.5.6) is verified directly by observing that

$$
\left[\frac{X}{2}, \frac{-Y-i Z+\mathrm{I} Z-\mathrm{I} i Y}{2}\right]=\mathrm{I} \frac{-Y-i Z+\mathrm{I} Z-\mathrm{I} i Y}{2} .
$$

These operators provide an easy way of going from one $K$ representation to another, and we call them "jumping operators" for this reason. We have that $v_{l j}$ is a multiple of

$$
\pi\left(L^{l-j}\left(J^{+}\right)^{l}\right) v_{00}
$$


In polar coordinates $z=r e^{i \alpha}$ we can write the operators as

$$
\pi(R)=e^{i \alpha}\left(2 i s r+i\left(r^{2}+1\right) \partial_{r}+\left(r-\frac{1}{r}\right) \partial_{\alpha}\right)
$$

and

$$
\pi(L)=e^{-i \alpha}\left(-2 i s r-i\left(r^{2}+1\right) \partial_{r}+\left(r-\frac{1}{r}\right) \partial_{\alpha}\right) .
$$

For the jumping operators we have

$$
\pi\left(J^{+}\right)=-e^{i \alpha}\left(\partial_{r}+\frac{i}{r} \partial_{\alpha}\right)
$$

and

$$
\pi\left(J^{-}\right)=-e^{-i \alpha}\left(\partial_{r}-\frac{i}{r} \partial_{\alpha}\right) .
$$

Finally for the last two operators of the basis $\left\{J^{+}, J^{-}, R, L, h, i h\right\}$ we have

$$
\pi(h)=2 s+2 r \partial_{r}
$$

and

$$
\pi(i h)=2 \partial_{\alpha}
$$

4.6 Normalizing operators. In this section we establish how the operators $J^{ \pm}, R, L$ defined above affect the $L^{2}$ norm of $v_{l j}$ and compute $v_{l j}$. It is well-known that

$$
\pi(R) v_{l j}=2 \sqrt{(l-j)(l+j+1)} v_{l, j+1}
$$

and

$$
\pi(L) v_{l j}=2 \sqrt{(l+j)(l-j+1)} v_{l, j-1} .
$$

In order to calculate the constants $C(l)$ from (4.5.4) we need to compute $v_{l l}$ and the constant for the intertwining operator.

Lemma 4.6.1. We have that $v_{l l}$ is a multiple of

$$
r^{l} e^{i l \alpha}\left(1+r^{2}\right)^{-s-l}
$$

Proof. Follows from solving the system of differential equations (4.4.1)-(4.4.2).

Lemma 4.6.2. The action of the intertwining operator $I: V_{s} \rightarrow V_{2-s}$ satisfies

$$
I . v_{l l}^{s}=(-1)^{l} \pi \frac{(\Gamma(s-1))^{2}}{\Gamma(l+s) \Gamma(s-l-1)} v_{l l}^{2-s} .
$$

Proof. It is clear that

$$
I . v_{l l}^{s}(r, \alpha)=\mathrm{const} \cdot v_{l l}^{2-s}(r, \alpha)
$$

since $I$ intertwines the action of $\pi_{s}$. The only question is establishing the value of the constant. We plug in $\alpha=0$ and apply $\left.\frac{d^{l}}{d r^{l}}\right|_{r=0}$ to both sides, recalling the definition of the intertwining operator (4.1.1). The result follows. 


\section{Lemma 4.6.3.}

$$
v_{l, \pm l}(z)=\frac{\sqrt{(-1)^{l} \Gamma(l+s) \Gamma(s-l-1)(2 l+1) !}}{l ! \pi \Gamma(s-1)} r^{l} e^{ \pm i l \alpha}\left(1+r^{2}\right)^{-s-l} .
$$

Proof. Let

$$
v_{l l}(r, \alpha)=b_{l} r^{l} e^{i l \alpha}\left(1+r^{2}\right)^{-s-l}
$$

for $b_{l}>0$. Then

$$
1=\left\langle v_{l l}, v_{l l}\right\rangle=\int_{\mathbf{C}} v_{l l}(z) \overline{I \cdot v_{l l}(z)} \frac{i}{2} d z \wedge d \bar{z}
$$

and from the previous Lemma we have

$$
1=\left|b_{l}\right|^{2}(-1)^{l} \pi \frac{(\Gamma(s-1))^{2}}{\Gamma(l+s) \Gamma(s-l-1)} \int_{r=0}^{\infty}\left(1+r^{2}\right)^{-s-l}\left(1+r^{2}\right)^{s-2-l} r d r .
$$

Evaluating the integral and solving for $b_{l}$ yields the desired result.

The resulting expressions for general $v_{l j}$ are given the next

Lemma 4.6.4. For $j \geqslant 0$ we have

$$
\begin{gathered}
v_{l j}(r, \alpha)=\frac{\sqrt{(-1)^{l} \Gamma(l+s) \Gamma(s-l-1)(2 l+1)(l-j) !(l+j) !}}{l ! \pi \Gamma(s-1)} \\
\frac{e^{i j \alpha}}{\left(r^{2}+1\right)^{s+l}} \sum_{k=0}^{l-j} r^{2(l-k)-j}\left(\begin{array}{c}
l \\
l-j-k
\end{array}\right)\left(\begin{array}{l}
l \\
k
\end{array}\right)(-1)^{k} .
\end{gathered}
$$

A similar formula holds for $j<0$.

Proof. This formula is verified by induction on $j$. The base case is $j=l$, which is Lemma 4.6.3. The induction step is going from $j$ to $j-1$; it is carried out by applying the operator $L$ from (4.5.12) with normalization from (4.6.2).

Lemma 4.6.4 above allows us to characterize the action of $\mathfrak{g}^{\mathbf{C}}$ on the $K$-types. By direct substitution we can verify that 


$$
\begin{aligned}
\frac{1}{2} \pi(h) v_{l j} & =\sqrt{\frac{(l+1-s)(l-1+s)\left(l^{2}-j^{2}\right)}{(2 l-1)(2 l+1)}} v_{l-1, j}-\sqrt{\frac{(l+2-s)(l+s)\left((l+1)^{2}-j^{2}\right)}{(2 l+1)(2 l+3)}} v_{l+1, j} \\
\pi\left(J^{+}\right) v_{l j} & =\sqrt{\frac{(l+2+j)(l+1+j)(s+l)(l+2-s)}{(2 l+1)(2 l+3)}} v_{l+1, j+1}-\sqrt{(l+j+1)(l-j)} v_{l, j+1}+ \\
& +\sqrt{\frac{(l-j)(l-j-1)(l-1+s)(l+1-s)}{(2 l-1)(2 l+1)}} v_{l-1, j+1} \\
\pi\left(J^{-}\right) v_{l j} & =\sqrt{\frac{(l+2-j)(l+1-j)(s+l)(l+2-s)}{(2 l+1)(2 l+3)}} v_{l+1, j-1}-\sqrt{(l-j+1)(l+j)} v_{l, j-1}+ \\
& +\sqrt{\frac{(l+j)(l+j-1)(l-1+s)(l+1-s)}{(2 l-1)(2 l+1)}} v_{l+1, j-1}
\end{aligned}
$$

\section{Automorphic MOdel}

Now we need to review some representation theory of $G$ in the automorphic model. All results are standard, except for formulae from Section 5.3 that are not to the best of our knowledge.

5.1 Spectral decomposition. Consider the decomposition of the right regular representation of $G$ on

$$
L^{2}(\Gamma \backslash G)=V_{\varphi_{0}} \oplus V_{\varphi_{1}} \oplus \cdots \oplus V_{\varphi_{d}} \oplus V_{\mathrm{temp}}
$$

Each $V_{\varphi_{n}}$ is isomorphic to a complementary series representation of $G$ with parameter $s_{n}$ and is irreducible; $V_{\text {temp }}$ is the reducible part consisting of the tempered representations (see $[8,20])$. Each $V_{\varphi_{n}}$ contains exactly one positive $K$-fixed vector, up to scalar multiplication; call this vector $\varphi_{n}$. These functions are well-defined on $\Gamma \backslash \mathbf{H}^{3}$, and satisfy

$$
\Delta \varphi_{n}+s_{n}\left(2-s_{n}\right) \varphi_{n}=0
$$

where $\Delta$ is the Laplace-Beltrami operator on the corresponding hyperbolic manifold.

5.2 Patterson-Sullivan theory. The function $\varphi_{0}$ has the smallest eigenvalue and is called the base eigenfunction; it can be realized explicitly as the integral of the Poisson kernel (raised to the power $\delta$ ) against the Patterson-Sullivan measure $\nu$. This measure is supported on a Cantor set $\Lambda \subset \partial \mathbf{H}^{3} \cong S^{2}$ and has Hausdorff dimension $\delta$ (see $[32,37]$ ). That is, the connection between $\nu$ and $\varphi_{0}$ is

$$
\varphi_{0}(\varphi, \theta, r)=\int_{(v, u) \in S^{2}}(P(\varphi, \theta, r ; v, u))^{\delta} d \nu(v, u)
$$


where

$$
P(\varphi, \theta, r ; v, u)=\frac{1-r^{2}}{1-2 r(\sin \theta \cos \varphi \sin u \cos v+\sin \theta \sin \varphi \sin u \sin v+\cos \theta \cos u)+r^{2}}
$$

is the Poisson kernel in the Euler angle parametrization. The variable $r$ is the distance in the disk model with corresponds to th $\frac{t}{2}$ in our coordinates on $A^{+}$.

We can also view the hyperbolic three space in the upper half-plane model $x_{1}+i x_{2}+j y$ with real $x_{1}, x_{2}, y$ as in [6]. The action of $G$ on $\mathbf{H}^{3}$ is by Möbius transformations with quaternion multiplication. In these coordinates we have

$$
\varphi_{0}\left(x_{1}+i x_{2}+j y\right)=\int_{z \in \mathbf{C} \cup\{\infty\}}\left(\frac{y\left(1+|z|^{2}\right)}{\left|z-\left(x_{1}+i x_{2}\right)\right|^{2}+y^{2}}\right)^{\delta} d \nu(z) .
$$

There is a similar statement relating eigenfunctions $\varphi_{n}$ with eigenvalues $s_{n}$ for $n \geqslant 1$ to certain distributions $D_{\Gamma, n}$, not measures, on $\partial \mathbf{H}^{3}$ via the Poisson-Helgason transform $[12,1]$ (see [13] for a discussion of the $\mathrm{SL}(2, \mathbf{R})$ case). We have

$$
\varphi_{n}(\varphi, \theta, r)=D_{\Gamma, n}\left((P(\varphi, \theta, r ; \cdot))^{s_{n}}\right) .
$$

In [12] it is proven that $D_{\Gamma, n}$ is in the dual space of $C^{2-s_{n}}\left(S^{2}\right)$.

5.3 Lie algebra elements in $K A^{+} K$ coordinates. We will subsequently need the action of $U\left(\mathfrak{g}^{\mathbf{C}}\right)$ in $K A^{+} K$ coordinates; specifically, in coordinates from (3.1.4). This equation is a smooth map from $\mathbf{R}^{6}$ to $G$. Its derivative is a map from $\operatorname{Lie}\left(\mathbf{R}^{6}\right)$ to $\mathfrak{g}$. The derivative map is invertible, and we can write the basis $\{h, i h, e, i e, f$, if $\}$ in terms of the basis $\left\{\partial_{\varphi}, \partial_{\theta}, \partial_{\psi}, \partial_{t}, \partial_{\theta_{2}}, \partial_{\varphi_{2}}\right\}$. We suppress the lengthy computation and only cite the final result.

$$
\begin{aligned}
\frac{h}{2}=-\operatorname{cth} t \sin \theta_{2} \partial_{\theta_{2}}+\cos \theta_{2} \partial_{t}-\operatorname{ctg} \theta & \operatorname{csch} t \sin \psi \sin \theta_{2} \partial_{\psi}+ \\
& +\cos \psi \operatorname{csch} t \sin \theta_{2} \partial_{\theta}+\operatorname{cosec} \theta \operatorname{csch} t \sin \psi \sin \theta_{2} \partial_{\varphi}
\end{aligned}
$$

$\frac{i h}{2}=\partial_{\varphi_{2}}$

$$
\begin{gathered}
\frac{e}{2}=-\frac{1}{2}\left(\operatorname{ctg} \theta_{2}+\operatorname{cth} t\right. \\
\left.+\frac{1}{8} e^{-t} \operatorname{cosec} \theta_{2}\right) \sin \varphi_{2} \partial_{\varphi_{2}}+\frac{\theta_{2}}{2} \operatorname{csch} t \sec \frac{\theta_{2}}{2}\left(\left(-1+\cos \varphi_{2}\right) \sin \varphi_{2}+e^{2 t}\left(1+\cos \theta_{2}\right) \sin \varphi_{2}+\right. \\
\left.+2 e^{t} \operatorname{ctg} \theta\left(\cos \psi \sin \varphi_{2}+\cos \varphi_{2} \cos \theta_{2} \sin \psi\right) \sin \theta_{2}\right) \partial_{\theta_{2}}+\frac{1}{2} \cos \varphi_{2} \sin \theta_{2} \partial_{t}+ \\
+\frac{1}{2} \operatorname{csch} t\left(-\cos \varphi_{2} \cos \psi \cos \theta_{2}+\sin \varphi_{2} \sin \psi\right) \partial_{\theta}- \\
-\frac{1}{2} \operatorname{cosec} \theta \operatorname{csch} t\left(\cos \psi \sin \varphi_{2}+\cos \varphi_{2} \cos \theta_{2} \sin \psi\right) \partial_{\varphi}
\end{gathered}
$$




$$
\begin{aligned}
& \frac{i e}{2}=-\frac{1}{2} \cos \varphi_{2}\left(\operatorname{ctg} \theta_{2}+\operatorname{cth} t \operatorname{cosec} \theta_{2}\right) \partial_{\varphi_{2}}-\frac{1}{2}\left(1+\cos \theta_{2} \operatorname{cth} t\right) \sin \varphi_{2} \partial_{\theta_{2}}- \\
& -\frac{1}{2} \sin \varphi_{2} \sin \theta_{2} \partial_{t}+\frac{1}{2}\left(\cos \varphi_{2}\left(\operatorname{ctg} \theta_{2} \operatorname{cth} t+\operatorname{cosec} \theta_{2}+\cos \psi \operatorname{ctg} \theta \operatorname{csch} t\right)-\right. \\
& \left.-\cos \theta_{2} \operatorname{ctg} \theta \operatorname{csch} t \sin \varphi_{2} \sin \psi\right) \partial_{\psi}+\frac{1}{2} \operatorname{csch} t\left(\cos \psi \cos \theta_{2} \sin \varphi_{2}+\cos \varphi_{2} \sin \psi\right) \partial_{\theta}+ \\
& +\frac{1}{2} \operatorname{cosec} \theta \operatorname{csch} t\left(-\cos \varphi_{2} \cos \psi+\cos \theta_{2} \sin \varphi_{2} \sin \psi\right) \partial_{\varphi} \\
& \frac{f}{2}=\frac{1}{2}\left(\operatorname{ctg} \theta_{2}-\operatorname{cth} t \operatorname{cosec} \theta_{2}\right) \sin \varphi_{2} \partial_{\varphi_{2}}+\frac{1}{2} \cos \varphi_{2}\left(-1+\cos \theta_{2} \operatorname{cth} t\right) \partial_{\theta_{2}}+\frac{1}{2} \cos \varphi_{2} \sin \theta_{2} \partial_{t}+ \\
& +\frac{1}{8} e^{-t} \operatorname{cosec} \frac{\theta_{2}}{2} \operatorname{csch} t \sec \frac{\theta_{2}}{2}\left(e^{2 t}\left(-1+\cos \theta_{2}\right) \sin \varphi_{2}+\left(1+\cos \theta_{2}\right) \sin \varphi_{2}+\right. \\
& \left.+2 e^{t} \operatorname{ctg} \theta\left(\cos \psi \sin \varphi_{2}+\cos \varphi_{2} \cos \theta_{2} \sin \psi\right) \sin \theta_{2}\right) \partial_{\psi}+ \\
& +\frac{1}{2} \operatorname{csch} t\left(-\cos \varphi_{2} \cos \psi \cos \theta_{2}+\sin \varphi_{2} \sin \psi\right) \partial_{\theta}- \\
& -\frac{1}{2} \operatorname{cosec} \theta \operatorname{csch} t\left(\cos \psi \sin \varphi_{2}+\cos \varphi_{2} \cos \theta_{2} \sin \psi\right) \partial_{\varphi} \\
& \frac{\text { if }}{2}=-\frac{1}{2} \cos \varphi_{2}\left(\operatorname{ctg} \theta_{2}-\operatorname{cth} t \operatorname{cosec} \theta_{2}\right) \partial_{\varphi_{2}}+\frac{1}{2}\left(-1+\cos \theta_{2} \operatorname{cth} t\right) \sin \varphi_{2} \partial_{\theta_{2}}+ \\
& +\frac{1}{2} \sin \varphi_{2} \sin \theta_{2} \partial_{t}+\frac{1}{4} e^{-t} \operatorname{csch} t\left(-\cos \varphi_{2}\left(\operatorname{ctg} \theta_{2}+\operatorname{cosec} \theta_{2}\right)-\right. \\
& \left.-2 e^{t} \operatorname{ctg} \theta\left(\cos \varphi_{2} \cos \psi-\cos \theta_{2} \sin \varphi_{2} \sin \psi\right)+e^{2 t} \cos \varphi_{2} \operatorname{tg} \frac{\theta_{2}}{2}\right) \partial_{\psi}- \\
& +\frac{1}{2} \operatorname{csch} t\left(\cos \psi \cos \theta_{2} \sin \varphi_{2}+\cos \varphi_{2} \sin \psi\right) \partial_{\theta}+ \\
& +\frac{1}{2} \operatorname{cosec} \theta \operatorname{csch} t\left(\cos \varphi_{2} \cos \psi-\cos \theta_{2} \sin \varphi_{2} \sin \psi\right) \partial_{\varphi}
\end{aligned}
$$

These formulae will be useful later.

\section{DECAY OF MATRIX COEFFICIENTS}

In this section we state well-known results on decay of matrix coefficients. We refer the reader to [26], [41], [21], [14] for proofs.

Let $\omega=1-\Omega_{K} \in U\left(\mathfrak{g}^{\mathbf{C}}\right)$.

Lemma 6.1.1. Let $(\pi, V)$ be a tempered unitary representation of $G$. Then for any $K$-finite $w_{1}, w_{2} \in V$

$$
\left\langle\pi\left(k_{1} a_{t} k_{2}\right) \cdot w_{1}, w_{2}\right\rangle \ll t e^{-t} \sqrt{\operatorname{dim} \pi(K) \cdot w_{1}}\left\|w_{1}\right\|_{L^{2}} \cdot \sqrt{\operatorname{dim} \pi(K) \cdot w_{2}}\left\|w_{2}\right\|_{L^{2}} .
$$

Furthermore, for any $w_{1}, w_{2} \in V$ we have

$$
\left\langle\pi\left(k_{1} a_{t} k_{2}\right) . w_{1}, w_{2}\right\rangle \ll t e^{-t}\left\|\omega\left(w_{1}\right)\right\|_{L^{2}}\left\|\omega\left(w_{2}\right)\right\|_{L^{2}} .
$$


Lemma 6.1.2. Fix $1<s_{0}<2$. Let $(\pi, V)$ be a unitary representation of $G$ that does not weakly contain any complementary series representation $V_{s}$ for $s>s_{0}$. Then for any $K$-finite $w_{1}, w_{2} \in V$

$$
\left\langle\pi\left(k_{1} a_{t} k_{2}\right) \cdot w_{1}, w_{2}\right\rangle \ll e^{-s_{0} t} \sqrt{\operatorname{dim} \pi(K) \cdot w_{1}}\left\|w_{1}\right\|_{L^{2}} \cdot \sqrt{\operatorname{dim} \pi(K) \cdot w_{2}}\left\|w_{2}\right\|_{L^{2}} .
$$

Furthermore, for any $w_{1}, w_{2} \in V$ we have

$$
\left\langle\pi\left(k_{1} a_{t} k_{2}\right) \cdot w_{1}, w_{2}\right\rangle \ll e^{-s_{0} t}\left\|\omega\left(w_{1}\right)\right\|_{L^{2}}\left\|\omega\left(w_{2}\right)\right\|_{L^{2}} .
$$

\section{Proof of Theorem 2.1.2}

\subsection{Setup. Let}

$$
N(T)=\sum_{\gamma \in \Gamma,|\gamma|<T} Y_{a^{\prime} a ; b^{\prime} b c}(K(\gamma))
$$

Also define $f_{T}(g)$ on $G$ by

$$
f_{T}(g)=Y_{a^{\prime} a ; b^{\prime} b c}(K(g)) \chi_{|g|<T} .
$$

Let $F_{T}: \Gamma \backslash G \times \Gamma \backslash G \rightarrow \mathbf{C}$ be defined by

$$
F_{T}(g, h)=\sum_{\gamma \in \Gamma} f_{T}\left(g^{-1} \gamma h\right)
$$

Observe that $N(T)=F_{T}(e, e)$.

For a fixed parameter $\eta$ (to be chosen later depending on $T$ ) let $\psi: G \rightarrow \mathbf{R}^{+}$be a smooth function supported in a ball of radius $\eta$ about $e$ and having integral 1 with respect to the measure we fixed in (3.1.5). Let $\Psi: \Gamma \backslash G \rightarrow R^{+}$be defined by

$$
\Psi(x)=\sum_{\gamma \in \Gamma} \psi(\gamma x)
$$

Let

$$
H(T)=\int_{\Gamma \backslash G \Gamma \backslash G} \int_{\Gamma} F_{T}(g, h) \Psi(h) \Psi(g) d h d g .
$$

\section{Lemma 7.1.1.}

$$
|H(T)-N(T)| \ll(a+1)^{3 / 2}\left(a^{\prime}+1\right)^{3 / 2} T^{2 \delta} \eta .
$$

Proof. The left hand side of (7.1.2) is at most

$$
\sum_{\gamma \in \Gamma} \int_{g \in \Gamma \backslash G} \int_{h \in \Gamma \backslash G}\left|f_{T}\left(g^{-1} \gamma h\right)-f_{T}(\gamma)\right| \Psi(g) \Psi(h) d h d g .
$$

We distinguish three cases.

1. If $|\gamma|>\frac{T}{1-\eta}$, then both $f_{T}\left(g^{-1} \gamma h\right)$ and $f_{T}(\gamma)$ vanish. 
2. If $\frac{T}{1-\eta} \geqslant|\gamma|>\frac{T}{1+\eta}$, then trivially

$$
\left|f_{T}\left(g^{-1} \gamma h\right)-f_{T}(\gamma)\right| \ll\left\|Y_{a^{\prime} a ; b^{\prime} b c}\right\|_{L^{\infty}} \ll \sqrt{(a+1)\left(a^{\prime}+1\right)} .
$$

3. Finally if $|\gamma| \leqslant \frac{T}{1+\eta}$, then

$$
\left|f_{T}\left(g^{-1} \gamma h\right)-f_{T}(\gamma)\right| \ll \eta\left\|Y_{a^{\prime} a ; b^{\prime} b c}\right\|_{C^{1}} \ll \eta\left((a+1)\left(a^{\prime}+1\right)\right)^{3 / 2} .
$$

Thus the error (7.1.3) is at most

$$
\sqrt{(a+1)\left(a^{\prime}+1\right)} \sum_{\frac{T}{1-\eta} \geqslant|\gamma|>\frac{T}{1+\eta}} 1+\eta\left((a+1)\left(a^{\prime}+1\right)\right)^{3 / 2} \sum_{|\gamma| \leqslant \frac{T}{1+\eta}} 1 \ll \eta\left((a+1)\left(a^{\prime}+1\right)\right)^{3 / 2} T^{2 \delta}
$$

by Lax-Phillips [24] and Theorem 2.1.1 above. Specifically, we use the leading term of their main theorem:

$$
\sum_{\gamma \in \Gamma,|\gamma|<T} 1=\mathrm{const} \cdot T^{2 \delta}+O\left(T^{2 \delta-\sigma}\right)
$$

where $\sigma$ depends on the spectral gap for $L^{2}(\Gamma \backslash G)$.

The previous Lemma allows us to work with $H$ rather than with $N$, and the next Lemma massages $H$ into a more pleasant form.

Lemma 7.1.2. We have

$$
H(T)=\int_{g \in G} f_{T}(g)\langle\pi(g) \Psi, \Psi\rangle d g
$$

Proof. We compute

$$
\int_{g \in \Gamma \backslash G} \int_{h \in \Gamma \backslash G} F_{T}(g, h) \Psi(h) \Psi(g) d h d g=\sum_{\gamma \in \Gamma} \int_{g \in \Gamma \backslash G} \int_{h \in \Gamma \backslash G} f_{T}\left(g^{-1} \gamma h\right) \Psi(h) \Psi(g) d h d g .
$$

In the inner integral substitute $x=g^{-1} \gamma h$, whence $h=\gamma g x$. Using $\Gamma$-invariance of $\Psi$ we get

$$
\sum_{\gamma \in \Gamma} \int_{g \in \Gamma \backslash G} \int_{x \in g^{-1} \gamma(\Gamma \backslash G)} f_{T}(x) \Psi(g x) \Psi(g) d x d g .
$$

Now the result follows by combining the sum over $\gamma$ with the integral in $x$ and renaming the variables. 
7.2 Spectral decomposition. Write $\Psi=\Psi_{0}+\cdots+\Psi_{d}+\Psi_{\text {temp }}$ with each $\Psi_{n}$ being the orthogonal projection onto $V_{s_{n}}$, and $\Psi_{\text {temp }}$ is the orthogonal projection onto $V_{\text {temp }}$. For $\Psi_{\text {temp }}$ we use decay of tempered matrix coefficients. We have

$$
\left|\left\langle\pi\left(k_{1} a_{t} k_{2}\right) \Psi_{\text {temp }}, \Psi_{\text {temp }}\right\rangle\right| \ll t e^{-t}\left\|\Psi_{\text {temp }}\right\|_{W^{2,2}}^{2}
$$

from Lemma 6.1.1. The norm $W^{2,2}$ is the second order Sobolev norm; it includes the $L^{2}$ norm of the function as well as the $L^{2}$ norms of all derivatives of orders 1 and 2 . Thus we get

$$
\int_{g \in G} f_{T}(g)\left\langle\pi(g) \Psi_{\text {temp }}, \Psi_{\text {temp }}\right\rangle d g \ll\left\|Y_{a^{\prime} b^{\prime} c b a}\right\|_{L^{1}}\left\|\Psi_{\text {temp }}\right\|_{W^{2,2}}^{2} T^{2} \log T \ll \eta^{-10} T^{2} \log T .
$$

Since complementary series representations of $G$ are irreducible, $\left\langle\pi(g) \Psi_{s_{1}}, \Psi_{s_{2}}\right\rangle$ vanishes whenever $s_{1}$ and $s_{2}$ are distinct. For the same reason $\left\langle\pi(g) \Psi_{s_{1}}, \Psi_{\text {temp }}\right\rangle=0$.

To get the simple version of the Main Theorem (2.1.4) we use the decomposition $\Psi=$ $\Psi_{0}+\Psi^{\perp}$, where $\Psi_{0}$ is the projection onto $V_{s_{0}}$ and $\Psi^{\perp}$ is the projection onto its orthogonal complement $V_{s_{0}}^{\perp}$. In this setting we can use Lemma 6.1.2 to get

$$
\left|\left\langle\pi\left(k_{1} a_{t} k_{2}\right) \Psi^{\perp}, \Psi^{\perp}\right\rangle\right| \ll_{\varepsilon} e^{\left(s_{1}-2+\varepsilon\right) t}\left\|\Psi^{\perp}\right\|_{W^{2,2}}^{2} .
$$

Then we have

$$
\int_{g \in G} f_{T}(g)\left\langle\pi(g) \Psi^{\perp}, \Psi^{\perp}\right\rangle d g \ll\left\|Y_{a^{\prime} b^{\prime} c b a}\right\|_{L^{1}}\left\|\Psi^{\perp}\right\|_{W^{2,2}}^{2} T^{2 s_{1}+\varepsilon} \ll \eta^{-10} T^{2 s_{1}+\varepsilon},
$$

and $\Psi_{0}$ contributes to the main term.

7.3 $K$-type decomposition. It remains to consider the non-tempered part

$$
\sum_{n} \int_{g \in G} f_{T}(g)\left\langle\pi(g) \Psi_{n}, \Psi_{n}\right\rangle d g .
$$

Expand $\Psi_{n}$ in the orthonormal basis $\left\{v_{l j}\right\}$ for the corresponding representation:

$$
\Psi_{n}=\sum_{l j}\left\langle\Psi_{n}, v_{l j}\right\rangle v_{l j}
$$

Here we use $v_{l j}: \Gamma \backslash G \rightarrow \mathbf{C}$ to denote a basis in the automorphic model; note that matrix coefficients can be computed in any model, while evaluations $v_{l j}(g), g \in G$ must be done in the automorphic model. In $K A^{+} K$ coordinates as in (3.1.4) and (3.1.5) we get

$$
\begin{aligned}
& 4 \pi \sum_{\substack{l, j, l^{\prime}, j^{\prime} \\
K, M \backslash K \\
A^{+}, \Gamma \backslash G}} \int_{a^{\prime} a ; b^{\prime} b c}\left(k_{1} k_{2}\right) \chi_{\left|a_{t}\right|<T}\left\langle\Psi_{n}, v_{l j}\right\rangle\left\langle v_{l^{\prime} j^{\prime}}, \Psi_{n}\right\rangle \\
& v_{l j}\left(x a_{t} k_{2}\right) \overline{v_{l^{\prime} j^{\prime}}\left(x k_{1}^{-1}\right)} d x d a_{t} d k_{2} d k_{1} .
\end{aligned}
$$


Now $Y_{a^{\prime} b^{\prime} c b a}$ and $v_{l j}$ have the same $K$-transformation properties on the right. Using orthogonality of matrix coefficients for $K$ we get

$$
4 \pi \overline{Y_{a c}(e)} Y_{a^{\prime} c}(e) \frac{\left\langle\Psi_{n}, v_{a b}\right\rangle\left\langle v_{a^{\prime} b^{\prime}}, \Psi_{n}\right\rangle}{(2 a+1)\left(2 a^{\prime}+1\right)} \int_{t=0}^{2 \log T}\left\langle\pi\left(a_{t}\right) v_{a c}, v_{a^{\prime} c}\right\rangle d a_{t} .
$$

Finally using the fact that

$$
\left\langle\Psi_{n}, v_{a b}\right\rangle=v_{a b}(e)+O\left(\left|\nabla v_{a b}(e)\right| \eta\right)
$$

we get

$$
\begin{aligned}
& 4 \pi \overline{Y_{a c}(e)} Y_{a^{\prime} c}(e) \frac{\overline{v_{a b}(e)} v_{a^{\prime} b^{\prime}}(e)}{(2 a+1)\left(2 a^{\prime}+1\right)} \int_{t=0}^{2 \log T}\left\langle\pi\left(a_{t}\right) v_{a c}, v_{a^{\prime} c}\right\rangle d a_{t}+ \\
& \quad+O\left(\eta\left|\nabla v_{a b}(e)\right| \overline{Y_{a c}(e)} Y_{a^{\prime} c}(e) \frac{v_{a^{\prime} b^{\prime}}(e)}{(2 a+1)\left(2 a^{\prime}+1\right)} \int_{t=0}^{2 \log T}\left\langle\pi\left(a_{t}\right) v_{a c}, v_{a^{\prime} c}\right\rangle d a_{t}\right)+ \\
& +O\left(\eta\left|\nabla v_{a^{\prime} b^{\prime}}(e)\right| \overline{Y_{a c}(e)} Y_{a^{\prime} c}(e) \frac{\overline{v_{a b}(e)}}{(2 a+1)\left(2 a^{\prime}+1\right)} \int_{t=0}^{2 \log T}\left\langle\pi\left(a_{t}\right) v_{a c}, v_{a^{\prime} c}\right\rangle d a_{t}\right)+O(\eta) .
\end{aligned}
$$

The error depends on Lipschitz norms of $v_{a b}$ and $v_{a^{\prime} b^{\prime}}$; we will control their dependence on the indices in an indirect way later.

Remark 7.3.1. A similar expression can be found in Proposition 3.13 of [3], but the significance of the Lipschitz norm is overlooked. In Lemma 7.7.2 we show how to control these norms, and the same treatment will work in the case of [3].

7.4 Main term. In the computation of the main terms of the Theorem we deliberately avoid most complications by working out a skeletal form first and then filling in the constants as necessary.

Proposition 7.4.1. We have

$$
\begin{aligned}
& \sum_{\substack{\gamma \in \Gamma \\
|\gamma|<T}} Y_{a^{\prime} b^{\prime}}\left(k_{1}(\gamma)\right) \overline{Y_{a b}\left(k_{2}^{-1}(\gamma)\right)}=c_{0} T^{2 \delta}+c_{1} T^{2 s_{1}}+\cdots+c_{d} T^{2 s_{d}}+ \\
& +O\left(T^{2 \frac{10 \delta+1}{11}}(\log T)^{1 / 11}(a+1)^{15 / 11}\left(a^{\prime}+1\right)^{15 / 11}\right)+O(\ldots)
\end{aligned}
$$

for certain constants $c_{n}=c_{n}\left(a, b, a^{\prime}, b^{\prime}\right), 0 \leqslant n \leqslant d$; three dots stand for the error terms of equation (7.3.2) with $\eta$ as in equation (7.4.5) below. For $c \neq 0$ we have

$$
\sum_{\substack{\gamma \in \Gamma \\|\gamma|<T}} Y_{a^{\prime} a ; b^{\prime} b c}(K(\gamma))=O\left(T^{2 \frac{10 \delta+1}{11}}(\log T)^{1 / 11}(a+1)^{15 / 11}\left(a^{\prime}+1\right)^{15 / 11}\right) .
$$


Lemma 7.4.2. With notation as before the asymptotics for matrix coefficients of $\left(\pi_{s}, V_{s}\right)$ are given by

$$
\left\langle\pi\left(a_{t}\right) v_{a c}^{s}, v_{a^{\prime} c}^{s}\right\rangle=\operatorname{const}\left(e^{t(s-2)-|c| t}+O\left(\left(a^{2}+a^{\prime 2}+1\right) e^{-s t-|c| t}\right)\right) .
$$

Proof. Without loss of generality take $c \geqslant 0$. From Lemma (4.6.4) we have

$$
v_{a c}(r, \alpha)=P_{2 a-c, c}(r)\left(1+r^{2}\right)^{-s-a}
$$

for a polynomial $P_{2 a-c, c}$. The powers appearing in the polynomial have the same parity; the lowest and the highest powers are $c$ and $2 a-c$, respectively. Combining $P_{2 a-c, c}\left(r e^{t}\right)$ and $P_{2 a^{\prime}-c, c}(r)$ we can write the matrix coefficient as

$$
e^{t s} \int_{r=0}^{\infty} \frac{Q_{a+a^{\prime}-c, c}\left(r^{2} ; e^{t}\right)}{\left(r^{2} e^{2 t}+1\right)^{a}\left(r^{2}+1\right)^{a^{\prime}}}\left(\frac{r^{2}+1}{r^{2} e^{2 t}+1}\right)^{s-2} \frac{r d r}{\left(r^{2} e^{2 t}+1\right)^{2}} .
$$

$Q$ is a polynomial with indicated maximal and minimal powers of $r^{2}$, and its coefficients depend on $e^{t}$ as inherited from the product $P_{2 a-c, c}\left(r e^{t}\right) \cdot P_{2 a^{\prime}-c, c}(r)$. It is natural to substitute $u=r^{2}$, followed by

$$
w=\frac{u+1}{u e^{2 t}+1} .
$$

These give

$$
\begin{aligned}
& e^{t s} \int_{w=1}^{e^{-2 t}} \frac{Q_{a+a^{\prime}-c, c}\left(\frac{1-w}{w e^{2 t}-1} ; e^{t}\right)}{\left(\frac{1-e^{-2 t}}{w-e^{-2 t}}\right)^{a}\left(\frac{e^{2 t}-1}{w e^{2 t}-1}\right)^{a^{\prime}} w^{a^{\prime}}} w^{s-2} \frac{d w}{1-e^{2 t}}= \\
& =\frac{e^{t s}}{\left(e^{2 t}-1\right)^{a+a^{\prime}+1}} \int_{1}^{e^{-2 t}} w^{s-2-a^{\prime}} Q_{a+a^{\prime}-c, c}\left(\frac{1-w}{w e^{2 t}-1} ; e^{t}\right)\left(w e^{2 t}-1\right)^{a+a^{\prime}} d w
\end{aligned}
$$

ignoring absolute constants. Now the integrand is a finite sum of powers of $w$ and can be easily evaluated once $Q$ is given. In particular it follows that the main term is of the form const $\cdot e^{t s-2 t-c t}$ and that the next highest term is $O\left(\left(a^{2}+a^{2}+1\right) e^{-s t-c t}\right)$ from 4.6.4, as desired.

Proof of Proposition 7.4.1. It is enough to extract the correct power of $T$ from the integral

$$
\int_{t=0}^{2 \log T}\left\langle\pi\left(a_{t}\right) v_{a c}, v_{a^{\prime} c}\right\rangle d a_{t}
$$

from (7.3.2) and to collect the error terms. Using the previous Lemma and the Haar measure on $A^{+}$from (3.1.3) we have that

$$
\int_{t=0}^{2 \log T}\left\langle\pi\left(a_{t}\right) v_{a c}, v_{a^{\prime} c}\right\rangle \operatorname{sh}^{2} t d t=\int_{t=0}^{2 \log T} e^{t s-|c| t} d t+O\left(T^{2(2-s)}\right) .
$$


Thus we get

$$
\sum_{n=0}^{d} c_{n} T^{2 s_{n}}
$$

for $c$ different from 0 as in the statement.

The error term in the Theorem comes from the error contributions (7.1.2), (7.3.2), and (7.2.1).

The optimal choice for $\eta$ that makes the errors from (7.1.2) and (7.2.1) equal:

$$
\eta=T^{\frac{2}{11}(1-\delta)}(\log T)^{1 / 11}(a+1)^{-\frac{3}{22}}\left(a^{\prime}+1\right)^{-\frac{3}{22}} .
$$

This gives the advertised error term in the statement.

We outline the calculation of the main term for the case $a=b=a^{\prime}=b^{\prime}=0$. Plugging these values into (7.3.2) gives

$$
4 \pi \overline{v_{00}(e)} v_{00}(e) \int_{t=0}^{2 \log T}\left\langle\pi\left(a_{t}\right) v_{00}, v_{00}\right\rangle d a_{t} .
$$

We have

$$
v_{00}(e)=\hat{\nu}(0,0)
$$

For the matrix coefficient we have

$$
\left\langle\pi\left(a_{t}\right) v_{00}, v_{00}\right\rangle=\frac{\operatorname{sh}(t \delta-t)}{(\delta-1) \operatorname{sh} t}
$$

which is computed in the line model using Lemma 4.6.3. The leading term (for large $t$ ) of this expression is

$$
\frac{e^{t \delta-2 t}}{\delta-1}
$$

Using the measure from (3.1.3) for the integral we get the leading term of

$$
\frac{\pi}{\delta(\delta-1)} \hat{\nu}(0,0) \overline{\hat{\nu}(0,0)} T^{2 \delta},
$$

as needed.

7.5 Conjugation invariance. Generalizing this computation seems very computationally intensive, so we use a trick to show that once the Theorem holds for the case of trivial spherical harmonics we have just considered, it holds in all cases. For $g \in G$, the spectral theory of $\Gamma \backslash G$ and $g^{-1} \Gamma g \backslash G$ is the same. So we consider the discrete group $g^{-1} \Gamma g$ and "linearize" in $g$. In essence we need to prove a multiplicity one statement for the coefficient of the main term by exploiting the transformation property under conjugation. We proceed inductively. The base case (constant spherical harmonics) was outlined above. The induction step is built on the next proposition.

Write inv : $G \rightarrow G$ for the inverse map. For functions $F_{1}, F_{2}, F_{3}$ on $K / M, A^{+}$, and $M \backslash K$, respectively, let

$$
F_{1} \otimes F_{2} \otimes F_{3}(g)=F_{1}\left(k_{1}(g)\right) F_{2}(a(g)) F_{3}\left(k_{2}(g)\right) .
$$


Proposition 7.5.1. Let $Y_{a a}$ and $Y_{a^{\prime} a^{\prime}}$ be a pair spherical harmonics, and suppose by induction that

$$
\lim _{T \rightarrow \infty} \frac{1}{T^{2 \delta}} \sum_{\gamma \in \Gamma} Y_{a^{\prime} a^{\prime}} \otimes \chi_{T} \otimes \overline{Y_{a a} \circ \operatorname{inv}}(\gamma)=\frac{\pi}{\delta(\delta-1)} \hat{\nu}_{\Gamma}\left(a^{\prime}, a^{\prime}\right) \overline{\hat{\nu}_{\Gamma}(a, a)} .
$$

Then

$$
\begin{array}{r}
\lim _{T \rightarrow \infty} \frac{1}{T^{2 \delta}} \sum_{\gamma \in \Gamma}\left(Y_{a^{\prime} a^{\prime}} \otimes \chi_{T} \otimes \overline{Y_{a+1, a+1} \circ \operatorname{inv}}(\gamma)+Y_{a^{\prime}+1, a^{\prime}+1} \otimes \chi_{T} \otimes \overline{Y_{a a} \circ \operatorname{inv}}(\gamma)\right)= \\
=\frac{\pi}{\delta(\delta-1)}\left(\hat{\nu}_{\Gamma}\left(a^{\prime}, a^{\prime}\right) \overline{\hat{\nu}_{\Gamma}(a+1, a+1)}+\hat{\nu}_{\Gamma}\left(a^{\prime}+1, a^{\prime}+1\right) \overline{\hat{\nu}_{\Gamma}(a, a)}\right) .
\end{array}
$$

Remark 7.5.2. In effect we suppose that the main term of Theorem 2.1.2 for one pair of spherical harmonics and for every group $\Gamma$, and prove that the main term is right for a new combination of pairs of spherical harmonics.

We need several lemmas to prove this Proposition.

Lemma 7.5.3. Let $\nu_{\Gamma}(z)$ be the Patterson-Sullivan measure for $\Gamma$ and let $g \in G$. Then, the Patterson-Sullivan measure for the conjugate of $\Gamma$ is

$$
\nu_{g^{-1} \Gamma g}(z)=P^{\delta}(g j, g z) \nu_{\Gamma}(g z) .
$$

Proof. This is immediate from the properties of the Patterson-Sullivan measure: the measure for the conjugated group is a combination of a push-forward with a Jacobian.

Lemma 7.5.4. We have

$$
\left.P^{\delta}\left(J^{+} j ; \varphi, \theta\right)\right|_{e \in G}=-\delta \sin \theta e^{i \varphi} .
$$

Proof. This is confirmed by direct calculation using (5.3.1)-(5.3.6) applied to the Poisson kernel (5.2.2) raised to the power $\delta$.

Proof of Proposition 7.5.1. Approximate the indicator $\chi_{T}$ in the definition of $f_{T}$ as follows. Let $\Xi_{T, A}^{+}$be 1 on $[0, T], 0$ on $[T+A, \infty)$, and interpolate linearly on $[T, T+A]$. Similarly let $\Xi_{T, A}^{-}$be 1 on $[0, T-A], 0$ on $[T, \infty)$, and interpolate linearly on $[T-A, T]$. We will choose $A<T$ later. We consider $\Xi^{+}$below; the treatment of $\Xi^{-}$is the same. We have

$$
\begin{aligned}
& \sum_{\gamma \in \Gamma} Y_{a^{\prime} b^{\prime}} \otimes \Xi_{T, A}^{+} \otimes \overline{Y_{a b} \circ \operatorname{inv}}\left(g^{-1} \gamma g\right)= \\
& =\frac{\pi}{\delta(\delta-1)} \hat{\nu}_{g^{-1} \Gamma g}\left(a^{\prime}, b^{\prime}\right) \overline{\hat{\nu}_{g^{-1} \Gamma g}(a, b)} T^{2 \delta}+c_{1} T^{2 s_{1}}+\cdots+c_{d} T^{2 s_{d}}+ \\
& \quad+O\left(T^{2 \frac{10 \delta+1}{11}}(\log T)^{1 / 11}(a+1)^{15 / 11}\left(a^{\prime}+1\right)^{15 / 11}\right)+O\left(T^{\delta-1} A\right) .
\end{aligned}
$$

Let $E>\varepsilon>0$ and let $g=\exp \varepsilon X$ for some $X \in \mathfrak{g}$. It is clear that all constants - both implied and the constants $c_{n}$ - are $1+O(E)$. 
Now we expand both sides in $\varepsilon$. The left hand side reads

$$
\begin{aligned}
\sum_{\gamma \in \Gamma}\left(Y_{a^{\prime} b^{\prime}} \otimes\right. & \Xi_{T, A}^{+} \otimes \overline{Y_{a b} \circ \operatorname{inv}}(\gamma)+ \\
& +\varepsilon Y_{a^{\prime} b^{\prime}} \otimes \Xi_{T, A}^{+} \otimes \overline{Y_{a b} \circ \operatorname{inv}}(\gamma X)+\varepsilon Y_{a^{\prime} b^{\prime}} \otimes \Xi_{T, A}^{+} \otimes \overline{Y_{a b} \circ \operatorname{inv}}(-X \gamma)+ \\
& \left.+O\left(\varepsilon^{2} 1 \otimes \Xi_{T, A}^{+} \otimes 1(\gamma)\right)\right) .
\end{aligned}
$$

The two $O(\varepsilon)$ terms are similar; we only treat the first one. As $t$ becomes large the only derivatives that contribute are

$$
\begin{aligned}
\frac{h}{2} & =\cos \theta_{2} \partial_{t}-\sin \theta_{2} \partial_{\theta_{2}} \\
\frac{i h}{2} & =\partial_{\varphi_{2}} \\
\frac{e}{2} & =\frac{1}{2} \cos \varphi_{2} \sin \theta_{2} \partial_{t}-\frac{1}{2}\left(\operatorname{ctg} \theta_{2}+\operatorname{cosec} \theta_{2}\right) \sin \varphi_{2} \partial_{\varphi_{2}}+\frac{1}{2} \cos \varphi_{2}\left(1+\cos \theta_{2}\right) \partial_{\theta_{2}} \\
\frac{i e}{2} & =-\frac{1}{2} \sin \varphi_{2} \sin \theta_{2} \partial_{t}-\frac{1}{2} \cos \varphi_{2}\left(\operatorname{ctg} \theta_{2}+\operatorname{cosec} \theta_{2}\right) \partial_{\varphi_{2}}-\frac{1}{2}\left(1+\cos \theta_{2}\right) \sin \varphi_{2} \partial_{\theta_{2}} \\
\frac{f}{2} & =\frac{1}{2} \cos \varphi_{2} \sin \theta_{2} \partial_{t}+\frac{1}{2}\left(\operatorname{ctg} \theta_{2}-\operatorname{cosec} \theta_{2}\right) \sin \varphi_{2} \partial_{\varphi_{2}}+\frac{1}{2} \cos \varphi_{2}\left(-1+\cos \theta_{2}\right) \partial_{\theta_{2}} \\
\frac{\text { if }}{2} & =\frac{1}{2} \sin \varphi_{2} \sin \theta_{2} \partial_{t}-\frac{1}{2} \cos \varphi_{2}\left(\operatorname{ctg} \theta_{2}-\operatorname{cosec} \theta_{2}\right) \partial_{\varphi_{2}}+\frac{1}{2}\left(-1+\cos \theta_{2}\right) \sin \varphi_{2} \partial_{\theta_{2}}
\end{aligned}
$$

It is enough to consider the case $X=J^{+}$. Here we have

$$
J^{+}=e^{i \varphi_{2}} \sin \theta_{2} \partial_{t}+i\left(\operatorname{ctg} \theta_{2}-\operatorname{cosec} \theta_{2}\right) e^{i \varphi_{2}} \partial_{\varphi_{2}}+e^{i \varphi_{2}}\left(-1+\cos \theta_{2}\right) \partial_{\theta_{2}} .
$$

Suppose $a=b$ and $a^{\prime}=b^{\prime}$. The effect of applying $J^{+}$is as follows. The first term turns the function

$$
Y_{a^{\prime} a^{\prime}} \otimes \Xi_{T, A}^{+} \otimes \overline{Y_{a a} \circ \operatorname{inv}}(\gamma)
$$

into

$$
-\frac{T}{2 A} Y_{a^{\prime} a^{\prime}} \otimes\left(\chi_{T+A}-\chi_{T}\right) \otimes \overline{Z_{a+1, a+1} \circ \operatorname{inv}}(\gamma),
$$

where $Z_{a+1, a+1}(\varphi, \theta)=Y_{a a}(\varphi, \theta) \sin \theta e^{i \varphi}=$ const $\cdot Y_{a+1, a+1}(\varphi, \theta)$. From (7.3.2) and Lemma 7.4.1 it follows that

$$
-\frac{T}{2 A} \sum_{\gamma \in \Gamma} Y_{a^{\prime} a^{\prime}} \otimes\left(\chi_{T+A}-\chi_{T}\right) \otimes \overline{Z_{a+1, a+1} \circ \operatorname{inv}}(\gamma)
$$

and

$$
-\delta \sum_{\gamma \in \Gamma} Y_{a^{\prime} a^{\prime}} \otimes \chi_{T} \otimes \overline{Z_{a+1, a+1} \circ \operatorname{inv}}(\gamma)
$$

have identical main terms (when $A \ll T$ ), so we use the second form. The other two terms of (7.5.3) turn the function (7.5.4) into

$$
-a Y_{a^{\prime} a^{\prime}} \otimes \chi_{T} \otimes \overline{Z_{a+1, a+1} \circ \mathrm{inv}}
$$


The net contribution from $J^{+}$is

$$
-(a+\delta) Y_{a^{\prime} a^{\prime}} \otimes \chi_{T} \otimes \overline{Z_{a+1, a+1} \circ \mathrm{inv}} .
$$

Now we compare to the right hand side. After linearizing in $\varepsilon$ we need to study two terms:

$$
\overline{\int Y_{a a}(\varphi, \theta) P^{\delta}\left(J^{+} j ; \varphi, \theta\right) d \nu_{\Gamma}(\varphi, \theta)}+\overline{\int Y_{a a}\left(-J^{+}(\varphi, \theta)\right) d \nu_{\Gamma}(\varphi, \theta)} .
$$

The action $-J^{+}(\varphi, \theta)$ is by a Möbius transformation on the boundary. Using Lemma 7.5.4 we rewrite the first term as

$$
-\delta \overline{\int Z_{a+1, a+1}(\varphi, \theta) d \nu_{\Gamma}(\varphi, \theta)} .
$$

Carrying out the calculation gives

$$
-a \overline{\int Z_{a+1, a+1}(\varphi, \theta) d \nu_{\Gamma}(\varphi, \theta)}
$$

for the second term. This matches the terms on the left-hand side. The term

$$
Y_{a^{\prime} a^{\prime}} \otimes \Xi_{T, A}^{+} \otimes \overline{Y_{a a} \circ \operatorname{inv}}(-X \gamma)
$$

is treated in a similar fashion.

Since $A$ and $\varepsilon$ were arbitrary, $O(\varepsilon)$ terms on both sides must match, whence the result.

7.6 $K$ invariance. The previous observation does not allow using completely general combinations of spherical harmonics due to symmetry, so we use $K$ invariance on one side to break this symmetry.

Lemma 7.6.1. Suppose have know that for a finite set $\mathcal{I}$

$$
\begin{aligned}
& \lim _{T \rightarrow \infty} \frac{1}{T^{2 \delta}} \sum_{\gamma \in \Gamma,|\gamma|<T} \sum_{\left(L, J, L^{\prime}, J^{\prime}\right) \in \mathcal{I}} Y_{L J}\left(k_{1}(\gamma)\right) \overline{Y_{L^{\prime} J^{\prime}}\left(k_{2}^{-1}(\gamma)\right)}= \\
&=\frac{\pi}{\delta(\delta-1)} \sum_{\left(L, J, L^{\prime}, J^{\prime}\right) \in \mathcal{I}} \hat{\nu}(L, J) \overline{\hat{\nu}\left(L^{\prime}, J^{\prime}\right) .}
\end{aligned}
$$

Then in fact

$$
\lim _{T \rightarrow \infty} \frac{1}{T^{2 \delta}} \sum_{\gamma \in \Gamma,|\gamma|<T} Y_{L J}\left(k_{1}(\gamma)\right) \overline{Y_{L^{\prime} J^{\prime}}\left(k_{2}^{-1}(\gamma)\right)}=\frac{\pi}{\delta(\delta-1)} \hat{\nu}(L, J) \overline{\hat{\nu}\left(L^{\prime}, J^{\prime}\right)}
$$

for each $\left(L, J, L^{\prime}, J^{\prime}\right) \in \mathcal{I}$. 
Proof. Redefine $f_{T}(g)$ from equation (7.1.1) by

$$
f_{T}(g)=\sum_{\left(L, J, L^{\prime}, J^{\prime}\right) \in \mathcal{I}} Y_{L J}\left(k_{1}(g)\right) \chi_{|g|<T} \overline{Y_{L^{\prime} J^{\prime}}\left(k_{2}^{-1}(g)\right)}
$$

and let $\varkappa \in K$. Consider the Theorem for the function $f_{T}(g \varkappa)$. We can carry out the proof as before until we get to (7.3.1). This equation will now read

$$
\begin{aligned}
4 \pi \sum_{\substack{L, J, L^{\prime}, J^{\prime}, J^{\prime \prime} \\
l, j, l^{\prime}, j^{\prime}}} \int_{\substack{K, M \backslash K K \\
A^{+}, \Gamma \backslash G}} Y_{L J}\left(k_{1}^{-1}\right) \overline{Y_{L^{\prime} J^{\prime \prime}}\left(k_{2}\right) \rho_{J^{\prime \prime} J^{\prime}}^{\left(L^{\prime}\right)}(\varkappa)} \chi_{\left|a_{t}\right|<T} \\
\\
\left\langle\Psi_{n}, v_{l j}\right\rangle\left\langle v_{l^{\prime} j^{\prime}}, \Psi_{n}\right\rangle v_{l j}\left(x a_{t} k_{2}\right) \overline{v_{l^{\prime} j^{\prime}}\left(x k_{1}^{-1}\right)} d x d a_{t} d k_{2} d k_{1}
\end{aligned}
$$

where $\rho$ is the corresponding representation of $K$. That is, each term in the sum transforms under $K$ in the same way as

$$
\overline{\delta(\delta-1)} \sum_{\left(L, J, L^{\prime}, J^{\prime}\right) \in \mathcal{I}} \hat{\nu}(L, J) \overline{\hat{\nu}\left(L^{\prime}, J^{\prime}\right)}
$$

transforms under $K$. Repeating this approach on the other side ensures that (7.6.1) holds term by term.

It is easy to see from Proposition 7.5.1 and Lemma 7.6.1 that the action of $U(\mathfrak{g})$ is rich enough to produce every pair of spherical harmonics from the one we checked by hand originally, whence the main terms of Theorem 2.1.2 are verified for all pairs of indices.

7.7 Bounds in the variables $a$ and $a^{\prime}$. We confirmed that $c_{0}\left(a, b, a^{\prime}, b^{\prime}\right)=\frac{\pi}{\delta(\delta-1)} \nu\left(Y_{a^{\prime} b^{\prime}}\right) \overline{\nu\left(Y_{a b}\right)}$. By applying the same differential operators $R, L, J^{ \pm}$to the Patterson-Sullivan distributions (5.2.3) we get that

$$
c_{n}\left(a, b, a^{\prime}, b^{\prime}\right)=\frac{\pi}{\delta(\delta-1)} D_{\Gamma, n}\left(Y_{a^{\prime} b^{\prime}}\right) \overline{D_{\Gamma, n}\left(Y_{a b}\right)} .
$$

Now the bound on the coefficients (2.1.2) in Theorem 2.1.2 follows from the regularity property of $D_{\Gamma, n}$ in Section 5.2 since $\left\|Y_{a b}\right\|_{L^{\infty}} \ll \sqrt{a+1}$.

Remark 7.7.1. A bound in terms of $a, a^{\prime}$ is also needed in [3], where Theorem 1.5 includes the incorrect claim that $c_{n}\left(a, a^{\prime}\right)$ are bounded for each $n$. Nevertheless it can be patched into a true statement

$$
\left|c_{n}\left(a, a^{\prime}\right)\right| \ll(|a|+1)^{1-s_{n}}\left(\left|a^{\prime}\right|+1\right)^{1-s_{n}} .
$$

For $\mathrm{SL}(2, \mathbf{R})$ regularity of automorphic distributions $D_{\Gamma, n}$ which we use here was analyzed by Schmid [33] for all representations and by Otal [31] for the complementary series representations. The results of [3] are little affected by this patch: it can only offset the error term slightly for bisector counts involving non-smooth functions.

Finally we need to establish a bound for $\nabla v_{a b}(e)$ that was needed in Proposition 7.4.1. We rely on Theorem 2.1.2, which has been established except for the power of $a+1, a^{\prime}+1$ in the error term; currently the error term depends on $\nabla v_{a b}(e)$. The main term has been confirmed, so we use it to prove the next 
Lemma 7.7.2. We have $\nabla v_{a b}^{s}(e) \ll(a+1)^{2-s}$ for $s=\delta$ and $\nabla v_{a b}^{s}(e) \ll(a+1)^{4-2 s}$ for $s \neq \delta$.

Proof. The action of $\mathfrak{g}$ on $v_{a b}$ is characterized by equations (4.6.1), (4.6.2), (4.6.4), (4.6.6), (4.6.5) above. It follows in particular that for any $X \in \mathfrak{g}$ of norm 1 we have

$$
\pi(X) v_{a b}=\sum_{i=-1}^{1} \sum_{j=-1}^{1} C_{i j} v_{a+i, b+j}
$$

with each $C_{i j} \ll a+1$. Therefore

$$
\pi(X) v_{a b}(e) \ll(a+1) \sum_{i=-1}^{1} \sum_{j=-1}^{1}\left|v_{a+i, b+j}(e)\right| .
$$

From the fact that the main term of Theorem 2.1.2 is correct we glean that

$$
D_{\Gamma, n}\left(Y_{a b}\right) \sim \text { const } \cdot v_{a b}(e)(a+1)^{s-1 / 2}
$$

for some absolute constant. The result follows.

This Lemma provides the control needed in Proposition 7.4.1, showing that the two error terms there are of the same order in $a$ and $a^{\prime}$. The proof of Theorem 2.1.2 in thus complete.

\section{Proof OF TheOREMS 2.2.1 AND 2.2.4}

8.1 Setup. Consider the sum

$$
S_{v, \Gamma}(T)=\sum_{\substack{\|\gamma v\|<T \\ \gamma \in \Gamma}} \chi_{G /(\operatorname{Stab} v \cap \Gamma)}(\gamma)
$$

with $v \neq 0$ a column vector in $\mathbf{R}^{4}$ in the cone $\{Q(v)=0\}$. Here $Q$ is a quadratic form of signature $(3,1)$, and assume that an isomorphism $\vartheta: \operatorname{PSL}(2, \mathbf{C}) \rightarrow \mathrm{SO}_{Q}^{\circ}(\mathbf{R})$ has been fixed. The norm $\|\cdot\|$ on $\mathbf{R}^{4}$ can be any norm whatsoever; in fact, it need not even satisfy the triangle inequality, but must have the scaling property, some non-vanishing, and continuity. For the Apollonian circle packing problem the form of interest is the Descartes form

$$
Q_{D}(a, b, c, d)=a^{2}+b^{2}+c^{2}+d^{2}-\frac{1}{2}(a+b+c+d)^{2}
$$

One can check that

$$
g \mapsto q g q^{-1}
$$

for

$$
q=\frac{1}{2}\left(\begin{array}{cccc}
1 & -1 & -1 & 1 \\
-1 & 1 & -1 & 1 \\
-1 & -1 & 1 & 1 \\
1 & 1 & 1 & 1
\end{array}\right)
$$


is an isomorphism $\mathrm{SO}\left(x^{2}+y^{2}+z^{2}-w^{2}\right) \cong \mathrm{SO}_{Q_{D}}(\mathbf{R})$. This map composed with $\iota$ from (3.1.1) defines the action of $\operatorname{PSL}(2, \mathbf{C})$ on $\mathbf{R}^{4}$. Let $\tilde{\Gamma}$ be the Apollonian group [21, 11]. It is generated by the matrices $S_{1}, S_{2}, S_{3}, S_{4}$ given by

$$
\left(\begin{array}{cccc}
-1 & 2 & 2 & 2 \\
& 1 & & \\
& & 1 & \\
& & & 1
\end{array}\right),\left(\begin{array}{lllll}
1 & & & \\
2 & -1 & 2 & 2 \\
& & 1 & \\
& & & 1
\end{array}\right), \quad\left(\begin{array}{lllll}
1 & & & \\
& 1 & & \\
2 & 2 & -1 & 2 \\
& & & 1
\end{array}\right),\left(\begin{array}{lllll}
1 & & & \\
& 1 & & \\
& & 1 & \\
2 & 2 & 2 & -1
\end{array}\right)
$$

inside $\mathrm{O}_{Q_{D}}(\mathbf{R})$. Let $\Gamma$ be the image of $\tilde{\Gamma}$ under the projection $\mathrm{O}_{Q_{D}}(\mathbf{R}) \rightarrow \mathrm{SO}_{Q_{D}}(\mathbf{R})$. Observe that these generators have determinant -1 , so that products of pairs of generators are in $\mathrm{SO}_{Q_{D}}(\mathbf{R})$. Thus we can count even length words in $\tilde{\Gamma}$ directly, and count odd length words by looking at the action of $\tilde{\Gamma}$ on $S_{1} v$. In what follows we consider only one of these sums as the treatment of the other sum is identical.

8.2 Region of summation. The set $G /(\operatorname{Stab} v \cap \Gamma)$ should be interpreted as one fundamental domain under the action of $\operatorname{Stab} v \cap \Gamma$ on $G$. It is known that Stab $v \cong N M$ since $v$ lies in the cone $\{Q=0\}$. Since the action of $\operatorname{SO}_{Q}(\mathbf{R})$ on the cone is transitive, there are $u \in \mathbf{R}^{4}$ and $g \in G$ so that $v=g u$ and Stab $u=N M$. Then we can rewrite the sum as

$$
S_{v, \mathrm{~B}}(T)=\sum_{\|g \beta u\|<T, \beta \in \mathrm{B}} \chi_{G /(\operatorname{Stab} u \cap \mathrm{B})}(\beta)
$$

with $\mathrm{B}=g^{-1} \Gamma g$. Now Stab $u=N M$, and by an argument from [21] $N M \cap \mathrm{B}=N \cap \mathrm{B}$. Therefore Stab $u \cap B$ is a discrete subgroup of $\mathbf{R}^{2}$ and can be isomorphic to the trivial group, $\mathbf{Z}$, or $\mathbf{Z}^{2}$. In the case of Apollonian circle packings only two of these options are possible, the trivial group and $\mathbf{Z}$. The first is realized by a bounded packing such as the one in Figure 1.1; the second corresponds to a periodic packing like the one in Figure 1.2.

Suppose the set $R=\operatorname{Stab} u \cap \mathrm{B}$ is non-trivial. If $G$ is written in Iwasawa coordinates $K A N$, then $R$ imposes a restriction only in the $N$ variable. That is, $R=K \cdot A \cdot \operatorname{Proj}_{N}(R)=$ $K$. $\operatorname{Proj}_{A N}(R)$. We denote these projections as $R$, too. The region is a strip bounded by a pair of parallel lines or the intersection of two such strips. In the upper half-space model $A N \cong \mathbf{H}^{3}$ this lifts to a region between two parallel vertical planes or the intersection of two such regions. Suppose without loss of generality that $j \in \mathbf{H}^{3}$ is within this region. Let $R_{1} \subset \mathbf{H}^{3}$ be the region below geodesics joining $j$ to the boundary of $\operatorname{Proj}_{N}(R) \subset \partial \mathbf{H}^{3}$. Clearly $R_{1} \subset R$; let $R_{2}$ be the complement of $R_{1}$ in $R$. It is easy to see that the region $R_{2}$ is contained within one fundamental domain for $\Gamma \subset G$. Therefore $R_{2}$ contains at most one point of $\Gamma$, whence

$$
S_{v, \mathrm{~B}}(T)=\sum_{\substack{\|\beta \beta u\|<\\ \beta \in \mathrm{B}}} \chi_{R_{1}}(\beta)+O(1) .
$$

The region $R_{1}$ conveniently replaces $R$ and is easy to parametrize in $K A^{+} K$ coordinates. It is left $K$-invariant, and in the right $K$ factor it is supported on directions emanating from $j$ which hit the boundary inside $\operatorname{Proj}_{N}(R)$. Thus $\chi_{R_{1}}(\beta)=\chi_{\operatorname{Proj}_{\partial \mathbf{H}^{3}} R}\left(k_{2}(\beta)\right)$. 
Now in the $K A^{+} K$ decomposition we compute that

$$
g \cdot k_{1} a_{t} k_{2} \cdot u=\frac{1}{2}\left(1+\cos \theta\left(k_{2}\right)\right)\left|a_{t}\right| \cdot g k_{1} u+O(1)
$$

The main term in this expression vanishes (i.e., $\cos \theta\left(k_{2}\right) \neq-1$ ) only if $k_{2} u$ is orthogonal to the highest weight vector in the $K$-representation. Write $\gamma=k_{1} a_{t} k_{2}$ for $\gamma \in \Gamma$. Then $\cos \theta\left(k_{2}(\gamma)\right)=-1$ for only finitely many $\gamma \in \Gamma$ since its action is properly discontinuous; therefore we tacitly assume $\cos \theta\left(k_{2}(\gamma)\right) \neq-1$.

8.3 Smoothing. To prepare the sum for an application of Theorem 2.1.2 we smooth the indicator $\chi_{R_{1}}$ to approximate from above and from below; let the smooth version $\chi_{R_{1}}^{U}$ be nonconstant in a neighborhood of size $U$ about the boundary of the region $S$. This introduces an error of $\ll U^{\delta} T^{\delta}$. Also take a partition of unity $\left\{\rho_{i}^{V}, i \in I\right\}$ subordinate to an open cover $\left\{V_{i} \mid i \in I\right\}$ with $\operatorname{diam}\left(V_{i}\right) \leqslant V$ for all $i \in I$; we can take $|I| \sim V^{-2}$. Using an obvious approximation argument we recast the sum as

$$
\sum_{i, j \in I} \sum_{\substack{\beta \in \mathrm{B} \\|\beta|<\frac{T}{\frac{1}{2}\left|1+\cos \theta\left(k_{2}(\beta)\right)\right|\left\|g k_{1}(\beta) u\right\|}}} \chi_{R_{1}}^{U}\left(k_{2}(\beta)\right) \rho_{i}^{V}\left(k_{1}(\beta)\right) \rho_{j}^{V}\left(k_{2}(\beta)\right) .
$$

\subsection{Applying Main Theorem. Fix}

$$
W=\max _{\operatorname{supp} \nu \cap R_{1}} f
$$

which exists since the intersection is compact. The function

$$
f\left(k_{2}\right)=\frac{1}{\frac{1}{2}\left(1+\cos \theta\left(k_{2}(\beta)\right)\right)}
$$

is unbounded, so we replace it by

$$
f\left(k_{2}\right) \wedge W
$$

and smooth it at the edge; call the resulting smooth function $\tilde{f}\left(k_{2}\right)$. First we consider the bounded function $\tilde{f}$ and then estimate the error committed in passing from $f$ to $\tilde{f}$.

Pick points $v_{i} \in V_{i}$ for each $i \in I$. Approximate the above sum by

$$
\sum_{i, j \in I} \sum_{\substack{\beta \in \mathrm{B} \\|\beta|<\frac{T \tilde{f}\left(v_{j}\right)}{\left\|g v_{i} u\right\|}}} \chi_{R_{1}}^{U}\left(k_{2}(\beta)\right) \rho_{i}^{V}\left(k_{1}(\beta)\right) \rho_{j}^{V}\left(k_{2}(\beta)\right) .
$$

The error incurred at this step is $\ll V T^{\delta}$.

From Theorem 2.1.2 in the simplified form this sum equals

$$
\sum_{i, j} \frac{\nu\left(\chi^{U} \rho_{j}^{V}\right) \nu\left(\rho_{i}^{V}\right)\left(\tilde{f}\left(k_{2}^{-1}\right)\right)^{\delta}}{\left\|g v_{i} u\right\|^{\delta}} T^{\delta}+O\left(T^{\frac{10 \delta+s_{1}}{11}} V^{-4-2\left(\frac{15}{11}+2\right)+\varepsilon}\right) .
$$


The error term comes from estimating Fourier coefficients of $\chi$ and $\rho$ and ensuring that the sum over $a, a^{\prime}$ converges. It is clear that the largest error contribution will come from the partitions of unity, so we can set $U=V$. Now the sum over $i$ and $j$ approximates the respective integral with error $\ll V T^{\delta}$. Thus the leading term becomes

$$
\frac{\pi}{\delta(\delta-1)}\left(\int_{\partial \mathbf{H}^{3}} \chi_{R_{1}}\left(k_{2}\right)\left(\frac{2}{1-\cos \theta\left(k_{2}\right)}\right)^{\delta} d \nu_{\mathrm{B}}\left(k_{2}\right) \int_{\partial \mathbf{H}^{3}} \frac{d \nu_{\mathrm{B}}\left(k_{1}\right)}{\left\|g k_{1} u\right\|^{\delta}}\right) T^{\delta}
$$

as in the statement of the Theorem; here we have used the fact that $f$ and $\tilde{f}$ only differ outside of the support of $\nu$. In the more common coordinates on $\partial \mathbf{H}^{3}=\mathbf{C} \cup \infty$ the first factor reads

$$
\int_{(\mathrm{B} \cap N) \backslash \partial \mathbf{H}^{3}}\left(|z|^{2}+1\right)^{\delta} d \nu_{\mathrm{B}}(z) .
$$

The error term comes from optimizing the two different error terms from this section. They are

$$
V T^{\delta}, T^{\frac{10 \delta+s_{1}}{11}} V^{-4-2\left(\frac{15}{11}+2\right)+\varepsilon} .
$$

Equating them gives $V=T^{-\frac{\delta-s_{1}}{129}}$, which works for all counting theorems stated above.

Now we estimate the error from bounding $f$. Sacrificing a constant factor we can focus on $k_{2}$ :

$$
\begin{aligned}
& \sum_{i, j \in I}\left(\sum_{|\beta|<\frac{T f\left(k_{2}(\beta)\right)}{\left\|g k_{1}(\beta) u\right\|}} \chi_{R_{1}}^{U}\left(k_{2}(\beta)\right) \rho_{i}^{V}\left(k_{1}(\beta)\right) \rho_{j}^{V}\left(k_{2}(\beta)\right)-\right. \\
& \left.-\sum_{|\beta|<\frac{T \tilde{f}\left(k_{2}(\beta)\right)}{\left\|g k_{1}(\beta) u\right\|}} \chi_{R_{1}}^{U}\left(k_{2}(\beta)\right) \rho_{i}^{V}\left(k_{1}(\beta)\right) \rho_{j}^{V}\left(k_{2}(\beta)\right)\right) \ll \\
& \ll \sum_{i \in I} \sum_{|\beta|<T f\left(k_{2}(\beta)\right)} \chi_{\{f>W\}}\left(k_{2}\right) \rho_{i}^{V}\left(k_{2}\right) \ll \sum_{i} \sum_{|\beta|<T f\left(k_{i}^{*}\right)} \chi_{\{f>W\}}\left(k_{2}\right) \rho_{i}^{V}\left(k_{2}\right),
\end{aligned}
$$

where $k_{i}^{*} \in V_{i}$ is a point where $f$ attains its maximum over $V_{i}$. Applying the Main Theorem in the simplified form gives

$$
\ll \sum_{i}\left(f\left(\left(k_{i}^{*}\right)^{-1}\right) T\right)^{\frac{10 \delta+s_{1}}{11}+\varepsilon} \sum_{a} a^{\frac{15}{11}+1}(a V)^{-l} .
$$

To ensure convergence in $a$ take $l>\frac{15}{11}+2$ and rewrite the expression as

$$
\left(V^{2} \sum_{i}\left(f\left(\left(k_{i}^{*}\right)^{-1}\right)\right)^{\frac{10 \delta+s_{1}}{11}+\varepsilon}\right) \cdot T^{\frac{10 \delta+s_{1}}{11}+\varepsilon} V^{-2-2-\frac{15}{11}} .
$$

The quantity in parentheses asymptotically is a finite integral since $f$ as a simple pole. The resulting error term is smaller than both error contributions in (8.4.1), so neglecting this term is justified. 
8.5 Ideal triangle case. Consider the function $N^{P}(T)$ for counting circles in an ideal triangle, not in the entire packing. Suppose that $v \in \mathbf{R}^{4}$ consists of the curvatures of the four largest circles in Figure 1.3. A circle from the packing that is contained in a particular ideal triangle is characterized by the property that the quadruple in which it appears has prescribed form in terms of generators (8.1.3). To wit, the first generator cannot be one that flips the smallest circle out of the triangle; say the circles are ordered so that this generator is $S_{4}$. The group $\left\{1, S_{4}\right\}$ acts on $G$ (and also on $K \backslash G=\mathbf{H}^{3}$ ) from the right; let $G_{4}$ be the fundamental domain for this action that lies under the hemisphere fixed by $S_{4}$. That is, $G_{4}$ contains the ideal triangle in Figure 1.3 by implicitly identifying $G_{4}$ with its projection onto $\mathbf{H}^{3}$ and $\partial \mathbf{H}^{3}$. The counting function $S_{v, \Gamma}(T)$ from (8.1.1) should be changed to

$$
S_{v, \Gamma}^{\Delta}(T)=\sum_{\substack{\|\gamma v\|<T \\ \gamma \in \Gamma}} \chi_{G_{4} /(\operatorname{Stab} v \cap \Gamma)}(\gamma)
$$

Proceeding as in the case of full packings we get the formula of Theorem 2.2.7 with $u$ and $\mathrm{B}$ defined as before.

\section{BIBLIOGRAPHY}

[1] Nalini Anantharaman and Steve Zelditch. Patterson-Sullivan distributions and quantum ergodicity. Ann. Henri Poincaré, 8(2):361-426, 2007.

[2] Alan F. Beardon and Bernard Maskit. Limit points of Kleinian groups and finite sided fundamental polyhedra. Acta Math., 132:1-12, 1974.

[3] Jean Bourgain, Alex Kontorovich, and Peter Sarnak. Sector estimates for hyperbolic isometries. Geometric and Functional Analysis, 20:1175-1200, September 2010.

[4] Jean Delsarte. Sur le gitter fuchsien. C. R. Acad. Sci. Paris, 214:147-179, 1942.

[5] W. Duke, Z. Rudnick, and P. Sarnak. Density of integer points on affine homogeneous varieties. Duke Mathematical Journal, 71:143-179, July 1993.

[6] J. Elstrodt, F. Grunewald, and J. Mennicke. Groups acting on hyperbolic space. Springer Monographs in Mathematics. Springer-Verlag, Berlin, 1998. Harmonic analysis and number theory.

[7] Alex Eskin and Curt McMullen. Mixing, counting, and equidistribution in Lie groups. Duke Mathematical Journal, 71:181-209, July 1993.

[8] I. M. Gel'fand, M. I. Graev, and I. I. Pjateckiı̌-Šapiro. Teoriya predstavlenii $i$ avtomorfnye funktsii. Generalized functions, No. 6. Izdat. "Nauka", Moscow, 1966.

[9] Anton Good. Local analysis of Selberg's trace formula, volume 1040 of Lecture Notes in Mathematics. Springer-Verlag, Berlin, 1983.

[10] Alexander Gorodnik and Amos Nevo. The ergodic theory of lattice subgroups, volume 172 of Annals of Mathematics Studies. Princeton University Press, Princeton, NJ, 2010. 
[11] Ronald L. Graham, Jeffrey C. Lagarias, Colin L. Mallows, Allan R. Wilks, and Catherine H. Yan. Apollonian circle packings: Geometry and group theory I. The Apollonian group. Discrete $\&$ Computational Geometry, 34:547-585, September 2005.

[12] Sandrine Grellier and Jean-Pierre Otal. Bounded eigenfunctions in the real hyperbolic space. Int. Math. Res. Not., (62):3867-3897, 2005.

[13] Sigurdur Helgason. Groups and geometric analysis, volume 83 of Mathematical Surveys and Monographs. American Mathematical Society, Providence, RI, 2000. Integral geometry, invariant differential operators, and spherical functions, Corrected reprint of the 1984 original.

[14] Roger E. Howe and Calvin C. Moore. Asymptotic properties of unitary representations. J. Funct. Anal., 32(1):72-96, 1979.

[15] Heinz Huber. Über eine neue Klasse automorpher Funktionen und ein Gitterpunktproblem in der hyperbolischen Ebene. I. Comment. Math. Helv., 30:20-62 (1955), 1956.

[16] Heinz Huber. Zur analytischen Theorie hyperbolischen Raumformen und Bewegungsgruppen. Math. Ann., 138:1-26, 1959.

[17] M. N. Huxley. Integer points, exponential sums and the Riemann zeta function. In Number theory for the millennium, II (Urbana, IL, 2000), pages 275-290. A K Peters, Natick, MA, 2002 .

[18] M. N. Huxley. Exponential sums and lattice points. III. Proc. London Math. Soc. (3), 87(3):591-609, 2003.

[19] Dubi Kelmer and Amir Mohammadi. Logarithm laws for one parameter unipotent flows. arXiv:1105.5325, May 2011.

[20] Anthony W. Knapp. Representation theory of semisimple groups. Princeton Landmarks in Mathematics. Princeton University Press, Princeton, NJ, 2001. An overview based on examples, Reprint of the 1986 original.

[21] Alex Kontorovich and Hee Oh. Apollonian circle packings and closed horospheres on hyperbolic 3-manifolds. J. Amer. Math. Soc., 24(3):603-648, 2011. With an appendix by Oh and Nimish Shah.

[22] Alex V. Kontorovich. The hyperbolic lattice point count in infinite volume with applications to sieves. Duke Math. J., 149(1):1-36, 2009.

[23] Steven P. Lalley. Renewal theorems in symbolic dynamics, with applications to geodesic flows, non-Euclidean tessellations and their fractal limits. Acta Math., 163(1-2):1-55, 1989.

[24] Peter D. Lax and Ralph S. Phillips. The asymptotic distribution of lattice points in Euclidean and non-Euclidean spaces. J. Funct. Anal., 46(3):280-350, 1982.

[25] Min Lee and Hee Oh. Effective circle count for apollonian packings and closed horospheres. 2011.

[26] François Maucourant. Homogeneous asymptotic limits of Haar measures of semisimple linear groups and their lattices. Duke Math. J., 136(2):357-399, 2007.

[27] Curtis T. McMullen. Hausdorff dimension and conformal dynamics. III. Computation of dimension. Amer. J. Math., 120(4):691-721, 1998. 
[28] Hee Oh and Nimish Shah. Counting visible circles on the sphere and kleinian groups. arXiv:1004.2129, April 2010.

[29] Hee Oh and Nimish Shah. Equidistribution and counting for orbits of geometrically finite hyperbolic groups. arXiv:1001.2096, January 2010.

[30] Hee Oh and Nimish Shah. The asymptotic distribution of circles in the orbits of kleinian groups. Inventiones Mathematicae, 187(1):1-35, 2012.

[31] Jean-Pierre Otal. Sur les fonctions propres du laplacien du disque hyperbolique. C. R. Acad. Sci. Paris Sér. I Math., 327(2):161-166, 1998.

[32] S. J. Patterson. The limit set of a Fuchsian group. Acta Math., 136(3-4):241-273, 1976.

[33] Wilfried Schmid. Automorphic distributions for $\mathrm{SL}(2, \mathbf{R})$. In Conférence Moshé Flato 1999, Vol. I (Dijon), volume 21 of Math. Phys. Stud., pages 345-387. Kluwer Acad. Publ., Dordrecht, 2000 .

[34] A. Selberg. Harmonic analysis and discontinuous groups in weakly symmetric Riemannian spaces with applications to Dirichlet series. J. Indian Math. Soc. (N.S.), 20:47-87, 1956.

[35] R. Sharp. Sector estimates for Kleinian groups. Port. Math. (N.S.), 58(4):461-471, 2001.

[36] Dennis Sullivan. The density at infinity of a discrete group of hyperbolic motions. Inst. Hautes Études Sci. Publ. Math., (50):171-202, 1979.

[37] Dennis Sullivan. Entropy, Hausdorff measures old and new, and limit sets of geometrically finite Kleinian groups. Acta Math., 153(3-4):259-277, 1984.

[38] J. G. van der Corput. Zum Teilerproblem. Math. Ann., 98(1):697-716, 1928.

[39] R. C. Vaughan. The Hardy-Littlewood method, volume 125 of Cambridge Tracts in Mathematics. Cambridge University Press, Cambridge, second edition, 1997.

[40] R. C. Vaughan and T. D. Wooley. Waring's problem: a survey. In Number theory for the millennium, III (Urbana, IL, 2000), pages 301-340. A K Peters, Natick, MA, 2002.

[41] Akshay Venkatesh. Sparse equidistribution problems, period bounds and subconvexity. Ann. of Math. (2), 172(2):989-1094, 2010.

Mathematics Department, Princeton University, Princeton, NJ

Email: ivinogra@math.princeton.edu 